\title{
Aerothermal Anchoring of CBAERO Using High Fidelity CFD
}

\author{
David J. Kinney 1 \\ NASA Ames Research Center, Moffett Field, Ca. 94035
}

The Configuration Based Aerodynamics (CBAERO) software package is used to predict the convective and radiative heating environments for the Crew Exploration Vehicle (CEV). A limited number of high fidelity CFD solutions are used to 'anchor' the engineering level estimates obtained using CBAERO.

\section{Introduction}

The Configuration Based Aerodynamics (CBAERO) software package, described in reference 1, is an engineering level aero-thermodynamics tool for predicting the aerodynamic and aero-thermodynamic environments of general vehicle configurations. CBAERO is based on accepted engineering level analysis for the estimation of both aerodynamic and aerothermal environments. In this paper a new anchoring process is described wherein high fidelity CFD results are used to 'anchor' the engineering level analyses. The anchoring process permits a few, select, CFD solutions to be used to beyond the specific flight condition they were original run at, allowing for the cost effective use of high fidelity, and computationally expensive, CFD solutions early in the design process when the vehicle trajectories are often in a constant state of change.

\section{CBAERO}

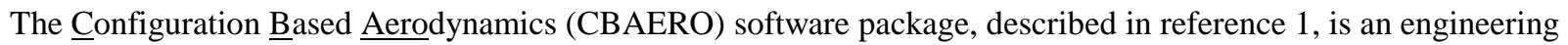
level aero-thermodynamics tool for predicting the aerodynamic and aero-thermodynamic environments of general vehicle configurations. For the present work CBAERO is used as the basis of the engineering level aerothermal analysis upon which corrections, based on CFD, are applied.

\section{A. Geometry Definition}

CBAERO makes use of an unstructured surface grid of triangles to define the Outer Mold Line (OML) of the vehicle configuration. No volume mesh is required. Figure 1 presents the unstructured surface mesh used to define the Crew Exploration Vehicle (CEV) geometry. The surface mesh for the 1/2 body contains 5,396 triangles.

\section{B. Subsonic Mach Numbers}

For the subsonic Mach number range CBAERO makes use of a fast, multi-pole, unstructured panel code formulation. The linear, integral formulation for subsonic, incompressible flow is solved using a fast multi-pole method, sometimes generically referred to as a fast tree method $[3,4]$. An octree data structure is used to define the spatial distribution of the surface triangles (panels), and their relative proximity to one another. Using the octree information, approximate, multi-pole, expansions are built for entire regions of the of the solution domain. The solution of the linear system of equations is then performed in $\sim \mathrm{N}^{*} \log \mathrm{N}$ time, $[3,4]$ using a preconditioned GMRES algorithm.

\section{Super and Hypersonic Mach Numbers}

For super through hypersonic Mach numbers the inviscid solution is based on independent panel methods, such as Modified-Newtonian, Tangent Cone, or Tangent Wedge formulations. All results presented here made use of the Modified-Newtonian formulation. Figure 2 depicts the surface pressure contours, using a Modified-Newtonian formulation, on the CEV configuration.

\footnotetext{
${ }^{1}$ Aerospace Engineer, Senior Member AIAA
} 
The independent panel methods provide the pressure at each surface triangle. The entropy on each triangle is taken equal to the post normal shock entropy. The known value of pressure and entropy at each panel fixes the thermodynamic state at the edge of the boundary layer. A table lookup procedure with data created using the Gordon and McBride (CEA) code, of reference [8] is used to calculate the remaining thermodynamic properties such as temperature and enthalpy.

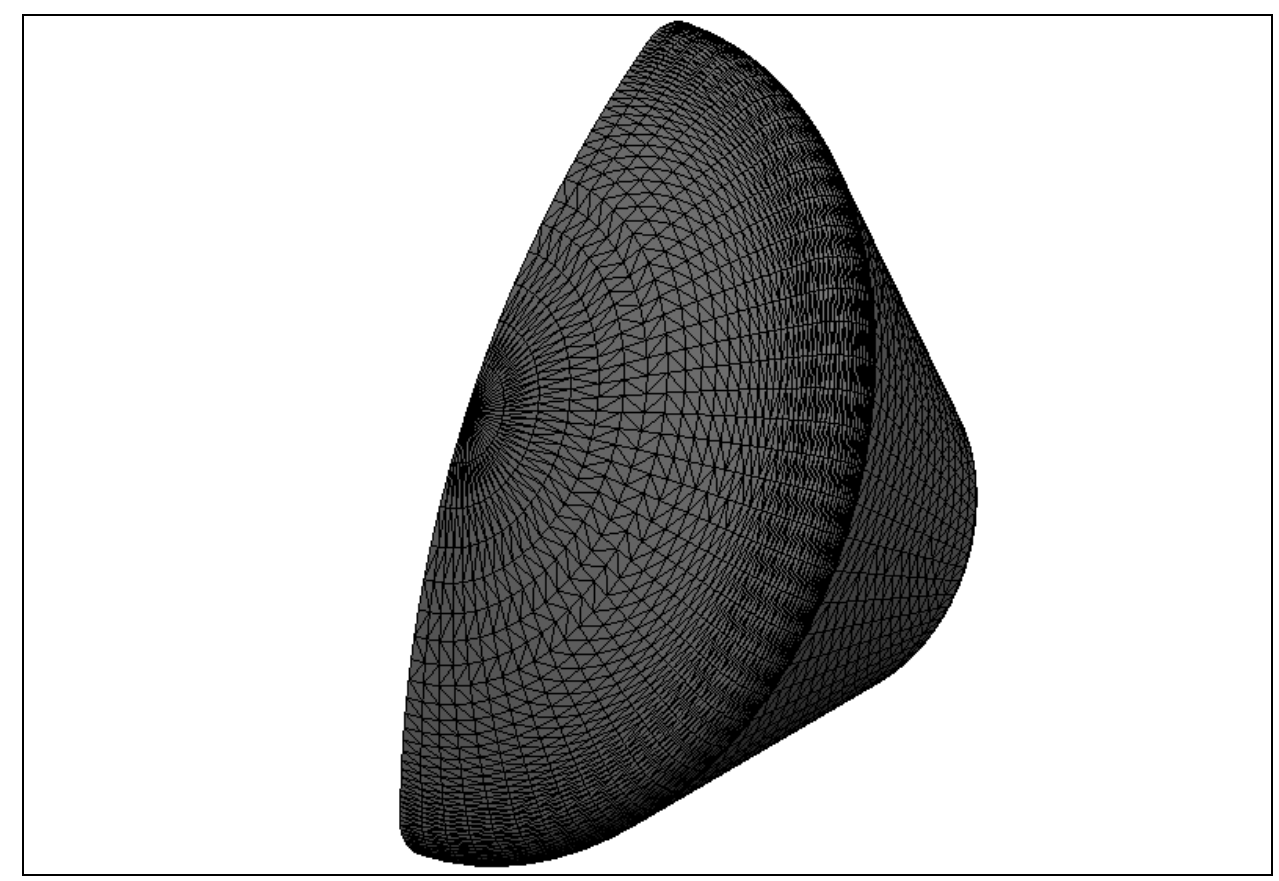

Fig. 1 Unstructured Triangulated Surface for the CEV, containing 5396 triangles.

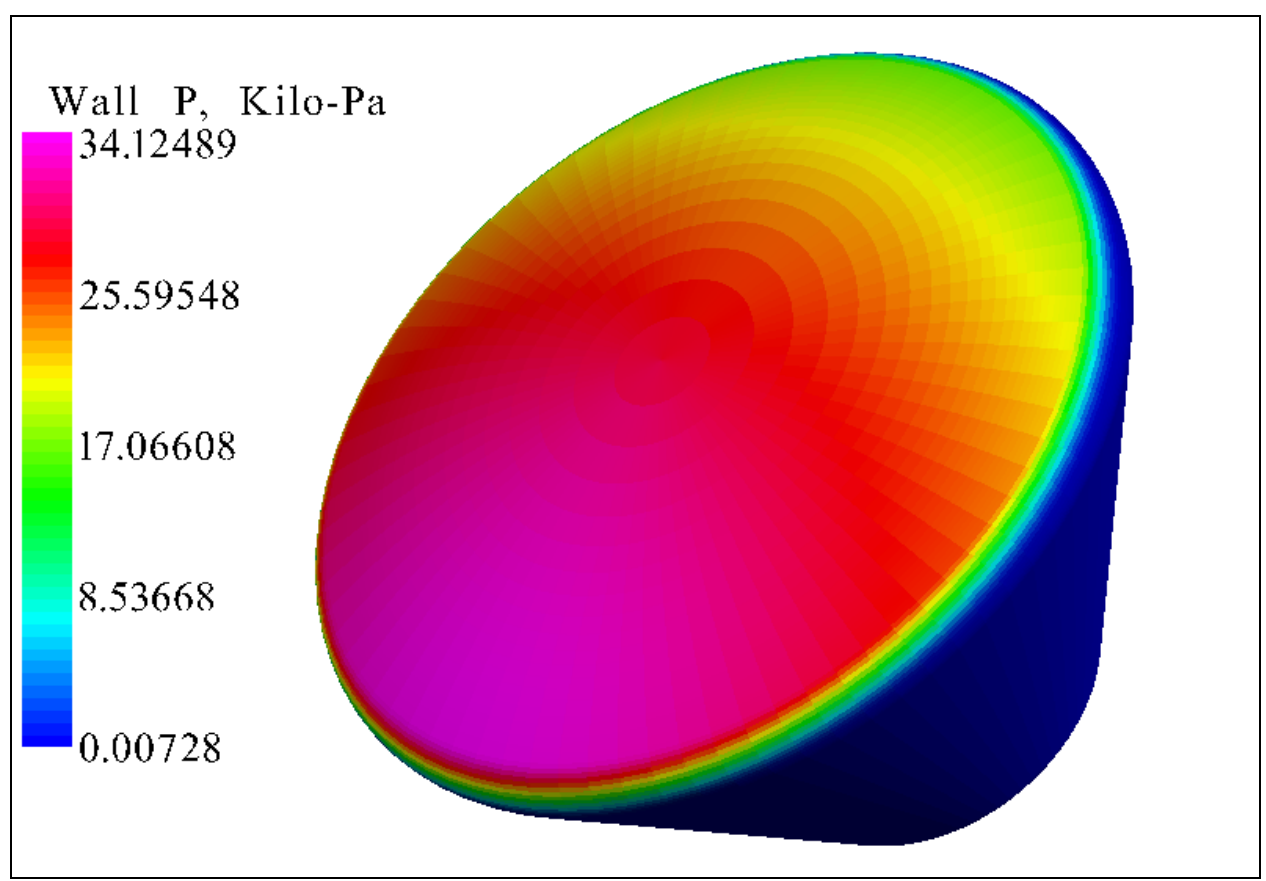

Fig. 2 Mach 32.2, $157.0^{\circ}$ Angle of Attack, CBAERO Surface Pressure Field. 


\section{Surface Streamline Pattern}

For subsonic flows the panel solution provides the local surface velocity. For supersonic and hypersonic flows the following approximation for the surface velocity field is used:

$$
\vec{V}_{\text {surface }}=\hat{n} \times \vec{V}_{\infty} \times \hat{n}
$$

Here $\hat{n}$ is the local surface normal, and $\vec{V}_{\infty}$ is the free stream velocity vector. This approximation is used to define the entire surface velocity flow field.

A robust integration algorithm is used to trace the streamline pattern over the entire vehicle. The algorithm calculates the streamline pattern in reverse, starting at the rear of the vehicle and integrating forward, until the stagnation point is reached. The algorithm continues to generate streamlines until every node/triangle in the mesh has a nearby streamline from which the distance to the stagnation point or an attachment line can be calculated.

The method of reference [2] is used to locate the attachment lines. The method makes use of topology considerations to locate attachment and separation lines. Here, only the attachments lines are of interest. The running lengths from either the stagnation point or the attachment lines are then calculated. These running lengths are used in the acreage heating models in the calculation of the local Reynolds number. Figure 3 depicts the streamlines on the $\mathrm{CEV}$, calculated using the above procedure for a free stream Mach number of 32.2 and an angle of attack of $157^{\circ}$.

\section{E. Blanked or Shadowed Regions}

CBAERO automatically calculates those regions that are 'blanked' or 'shadowed' by other windward facing surfaces. A sorting algorithm is used to quickly locate any windward facing surfaces that are 'blanked' by other windward facing surfaces. The pressure coefficient on blanked surfaces is set to an empirically derived value which tends to zero at high Mach numbers.

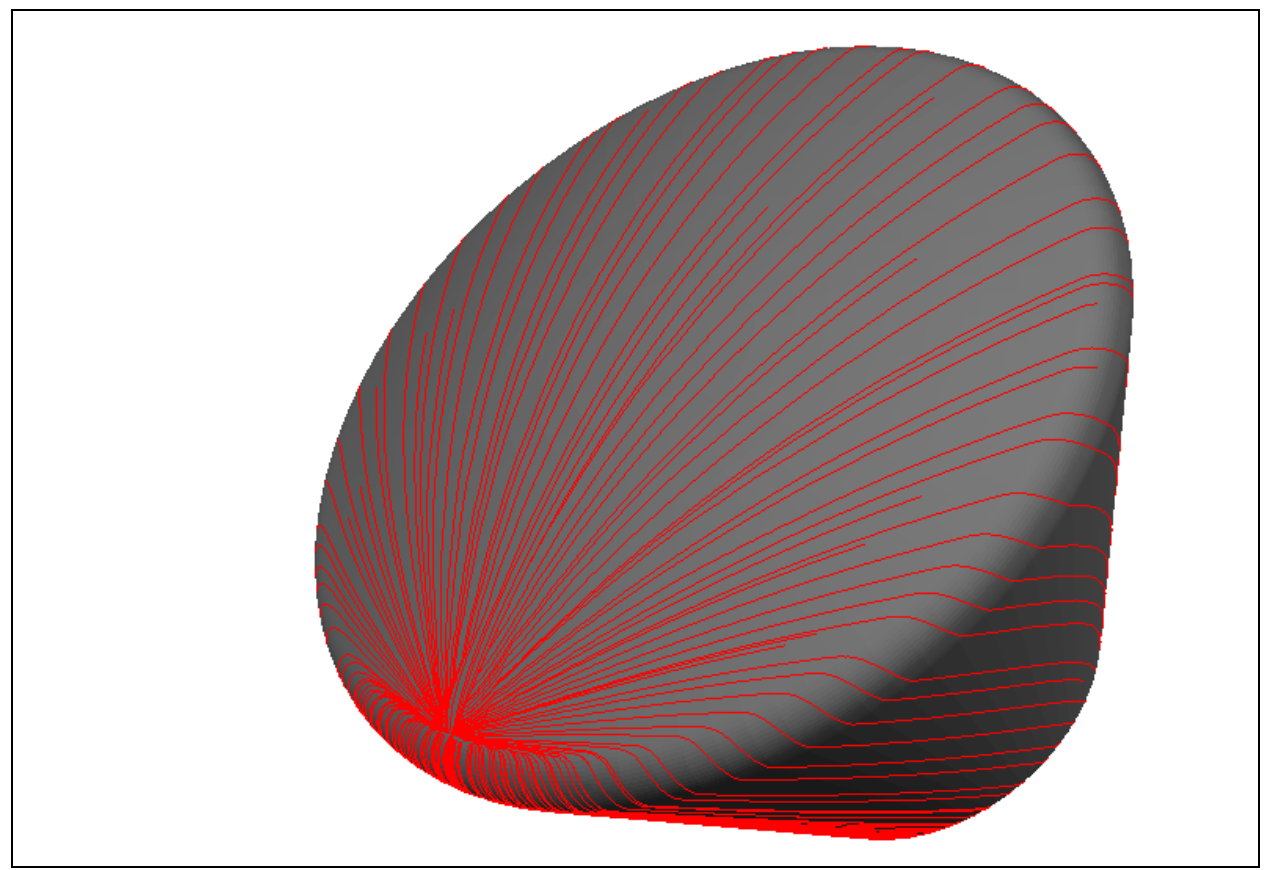

Fig. 3 Mach 32.2, 157.0 ${ }^{\circ}$ Angle of Attack, CBAERO Surface Streamline Pattern

\section{F. Stagnation Point Heating}

The convective heating correlation of Tauber [5] is used to predict the convective heating at the stagnation point. The correlation is driven by the stagnation edge conditions, as well as the local radius. The radius is estimated 
by fitting a general bi-cubic surface at the location of the stagnation point. Sufficient neighboring triangles are used to calculate a best fit surface, in a least squares sense. The primary curvatures are then calculated directly from this cubic surface. An average of the principle curvatures is used to determine the local radius. For a perfectly spherical stagnation point both principle curvatures will be identical and the local radius estimate will reduce to the expected radius of the sphere.

\section{G. Attachment Line Heating}

As with the stagnation point, the convective heating correlation of Tauber [5] is used to predict the convective heating along the attachment lines. The correlation is driven by the stagnation edge conditions, as well as the local radius. The radius is estimated by fitting a general bi-cubic surface at the location of each of the previously identified triangles lying on the attachment line. Sufficient neighboring triangles are used to calculate a best fit surface, in a least squares sense.

When fitting the bi-cubic patch, the two local surface coordinate directions are aligned parallel and normal to the local surface velocity. The third coordinate direction remains normal to the surface. The primary curvatures are then calculated directly from this cubic surface. The surface curvature in the direction normal to the local surface velocity is used to determine the local radius. For a simple swept cylinder this formulation would return the expected radius of the cylinder. This radius, along with an appropriate sweep correction is used in Tauber's correlation to predict the attachment line convective heating.

\section{H. Radiative Heating}

The Tauber-Sutton radiative heating correlation of reference [9] is used to predict the stagnation and off stagnation radiative heating environments. The correlation is of the form

$$
\dot{q}_{\text {radiative }}=C \cdot \rho^{A} R^{B} \cdot f(V)
$$

Here $\rho$ is the free stream density, $\mathrm{R}$ is the local radius at the stagnation point, $\mathrm{V}$ is the velocity normal to the local shock surface, $\mathrm{f}(\mathrm{V})$ is the intensity function, and $\mathrm{A}, \mathrm{B}$, and $\mathrm{C}$ are constants. The local radius, as determined for the convective heating model, is again used. The off stagnation point distribution is scaled from the stagnation point value with an empirically derived function that is a function of the local impact angle.

\section{Acreage Heating}

The acreage heating model is based on either laminar or turbulent flat plate reference enthalpy methods for the skin friction, and Reynold's analogy to estimate the Stanton number and hence the local heating rate. A simple Mangler correction is used to adjust the two-dimensional estimates. The running lengths, calculated using the streamline patterns, are used to estimate the local Reynolds number. For both laminar and turbulent flows a reference temperature, based on Eckert's method, is calculated:

$$
T_{\text {ref }}=0.5 \cdot\left(T_{e}+T_{w}\right)+0.11 \cdot R_{f} \cdot(\gamma-1) \cdot M_{e}^{2} \cdot T_{e}
$$

The recovery factor, $R_{f}$, is calculated as the square root of the Prandtl number for laminar flows, and the cube root of the Prandtl number for turbulent flows. The Prandtl number is evaluated at the reference temperature. For laminar flows the skin friction coefficient and Stanton number are calculated as:

$$
\begin{gathered}
C_{f}=0.664 / \sqrt{\mathrm{Re} / M_{f}} \cdot T_{e} / T_{r e f} \\
S_{t}=0.5 \cdot C_{f} \cdot \operatorname{Pr}^{-2 / 3}
\end{gathered}
$$

For turbulent flows the relations for skin friction and Stanton number are:

$$
\begin{gathered}
C_{f}=0.37 /\left[\log _{10}\left(\operatorname{Re} / M_{f}\right)\right]^{2.584} \cdot T_{e} / T_{r e f} \\
S_{t}=0.5 \cdot C_{f} \cdot \operatorname{Pr}^{-2 / 3}
\end{gathered}
$$

In the above relations, $M_{f}$ is the Mangler factor. For laminar flows, $M_{f}$ is equal to 3 and for turbulent flows it is set to 2. The enthalpy based film coefficient, recovery enthalpy, and convective heating are defined as:

$$
C_{H}=\rho_{r e f} \cdot U_{e} \cdot S t
$$




$$
\begin{aligned}
& H_{r e c}=H_{e}+0.5 \cdot R_{f} \cdot U_{e}^{2} \\
& \dot{Q}_{\text {conv }}=C_{H} \cdot\left(H_{r e c}-H_{w}\right)
\end{aligned}
$$

In the above relations, $H_{e}$ is the edge static enthalpy, $H_{r e c}$ is the recovery enthalpy, $U_{e}$ the edge velocity, and $H_{w}$ is the wall enthalpy.

The Reynolds number used above is based on the geometric running lengths estimated using the Newtonian surface velocity approximation for super or hypersonic flows or the fast-panel solution for subsonic flows. On windward surfaces the running length is taken directly as the geometric value, on leeward surface a weighted running length is used. The process is similar to that used in reference [17]. For both laminar and turbulent flows, the weighted reference length, $S_{w}$, is calculated as

$$
\rho_{e} \mu_{e} U_{e} S_{w}=\int_{0}^{S} \rho_{e} \mu_{e} U_{e} d S
$$

The integration is along a streamline. A smooth transition, from the geometric to the weighted running length is used as the streamline passes from the windward to leeward surface. Finally, it is noted that in the calculation of the local Reynolds number the reference density and viscosity are used.

\section{J. Transition}

CBAERO can be run fully laminar, fully turbulent, or transitional. For transitional flow a simple model, based on $\mathrm{Re}_{\theta} / \mathrm{M}_{\text {edge }}$ is used to determine the onset of transition. The transition length is set equal to the laminar run length, with a linear transition from laminar to fully turbulent flow.

\section{K. Free Molecular and Rarefied Flow}

CBAERO does include both a free molecular flow model [18] and bridging function methodology [19] for rarefied flows. However, for the present work only the above continuum models were used for the databases created for the CEV thus far.

\section{Catalytic Surface Model}

To date, all the aerothermal databases for the CEV have been run fully catalytic. However, CBAERO does include partially catalytic heating models. The partially catalytic heating models developed for CBAERO incorporate mass transfer coefficients and global surface reaction rates for a chemically frozen boundary layer [14]. The wall surface reaction rates are determined by the speeds at which oxygen and nitrogen atoms move towards the wall, which in turn depend on the wall temperatures and the atomic masses of Oxygen and Nitrogen. The wall reaction rate constants are calculated separately for Oxygen and Nitrogen. The recombination coefficients are normalized wall reaction rates which are found experimentally to be strong functions of surface temperatures and material characteristics [7].

A similarity variable, $\beta$, represents the velocity gradient at the stagnation point for a hemisphere. Calculated from Newtonian theory, it depends on the free stream velocity, nose radius and the free stream and boundary layer edge densities,

$$
\beta=\left(V_{\infty} / R_{\text {nose }}\right) \cdot \sqrt{2.0 * \rho_{\infty} / \rho_{\text {edge }}}
$$

For the acreage heating points it can be demonstrated that the similarity variables are the velocity at the boundary layer edge and the running length. The similarity variable is taken to be of the form

$$
\beta=C \cdot\left(V_{\infty} / X\right) \cdot f\left(S / R_{\text {nose }}\right)
$$

Where $\mathrm{C}$ is a constant dependent on the form of the laminar flat plate heating model, $\mathrm{X}$ is the local running length, and $f(S, R)$ is an exponentially decaying function fitted to smoothly match the flat plate catalytic surface model with the stagnation catalytic surface model.

The fraction of atoms that reach the wall and recombine is represented by $\phi_{\mathrm{O}, \mathrm{N}}$ which is calculated for both Oxygen and Nitrogen. The convective heat transfer rate for a fully catalytic surface can be written as: 


$$
\dot{q}_{\text {convective }}^{F C}=H_{i} \cdot\left(I_{R}^{F C}-I_{\text {wall }}\right)
$$

Here 'FC' stands for 'Fully Catalytic'.

It should be noted that the recovery enthalpy in a fully catalytic formulation, $I_{\mathrm{R}}^{\mathrm{FC}}$, contains the entire energy of the $\mathrm{O}$ and $\mathrm{N}$ atoms which have dissociated at the boundary layer edge. For the partially catalytic case, however, a fraction of these atoms, $\left(1.0-\phi_{\mathrm{O}, \mathrm{N}}\right)$, do not recombine on the surface. Therefore, the fully catalytic recovery enthalpy is replaced by the partially catalytic recovery enthalpy, $I_{\mathrm{R}}^{\mathrm{PC}}$, by subtracting the enthalpies of the atoms which do not recombine:

$$
I_{R}^{P C}=I_{R}^{F C}-\sum_{O, N}\left[\left(1-\phi_{O, N}\right) \cdot X_{O, N}^{M} \cdot I_{O, N}^{D}\right]
$$

Here 'PC' stands for 'Partially Catalytic', $X_{\mathrm{O}, \mathrm{N}}^{\mathrm{M}}$ represents the mass fractions of Oxygen and Nitrogen atoms at the boundary layer edge, and $I_{\mathrm{O}, \mathrm{N}}^{\mathrm{D}}$ are their heats of dissociation or recombination. These mass fractions are determined by the chemical equilibrium program CEA [8] at the boundary layer edge conditions and stored in a tabular fashion for fast lookup within CBAERO.

\section{Surface Energy Balance}

For any triangle on the surface an energy balance for a non-conducting wall is formulated. In general, the energy radiated from the surface must equal the sum of the convective and incident shock-radiative heating:

$$
\dot{q}_{\text {convective }}+\dot{q}_{\text {radiative }}=\sigma \cdot \varepsilon \cdot T_{\text {wall }}^{4}
$$

Since the convective heating depends on the final wall temperature this non-linear relationship must be solved iteratively at each triangle.

\section{CFD}

To judge the accuracy of CBAERO, comparisons have been made to the NASA Ames CFD codes DPLR and NEQAIR. DPLR [10] is a parallel multi-block finite volume code that solves the reacting Navier-Stokes equations including finite-rate chemistry and the effects of thermal non-equilibrium. Based on a finite-volume implementation of a modified Steger-Warming flux splitting method with Gauss-Seidel line relaxation, DPLR is a truly parallel, multi-block codeNominal third-order spatial accuracy, for inviscid fluxes, is obtained via MUSCL extrapolation coupled with a minmod limiter. Viscous fluxes are computed to second-order spatial accuracy using central differencing.

NEQAIR [11] is a line-by-line spectroscopy and one-dimensional radiation transport tool. The code computes radiation transport (from the free stream to the vehicle surface) along straight lines of sight using a tangent-slab approximation. The data at points on a line of sight are obtained through interpolation from a volume solution computed using DPLR, i.e., radiative heating is obtained a posteriori using converged flow solutions, essentially assuming the flow and radiative processes are uncoupled. NEQAIR constructs accurate spectra at every point on a line of sight, computes the integrated radiative intensity from the spectral distribution, and transports the energy to the next point on the line of sight through one-dimensional transport.

\section{A. Comparison of CBAERO to CFD}

In figures 4 through 8 CBAERO results for a Mach 28.4, $152^{\circ}$ angle of attack, laminar case are compared with results from the NASA Ames CFD codes DPLR and NEQAIR [10, 11]. For this case, the CBAERO results took 3.7 seconds of CPU time on a 2.26 Pentium M laptop.

The CBAERO pressure distribution presented in figure 4 compares favorably with the DPLR result. Similarly the CBAERO prediction for convective heating is presented in figure 5. The CBAERO results compare nicely with the DPLR results, slightly under predicting the peak shoulder heating by approximately $10 \%$.

Figure 6 compares the CBAERO temperature distribution with the DPLR highlighting the details of the leeward heating environment. Once again, CBAERO does a reasonable job of predicting the leeward heating environment. 
Figure 7 compares the CBAERO and DPLR predictions for the laminar shear magnitude. CBAERO clearly under predicts the peak shear stress near the shoulder, but otherwise predicts the general shape of the shear stress distribution.

Figure 8 compares the CBAERO and NEQAIR [11] results for the radiative heating. The peak radiative heating magnitude compares favorably with the NEQAIR results, with the peak slightly offset from the NEQAIR results. The over all shape of the radiative heating distribution is generally captured.

Figures 9 and 10 compare the surface distribution of the CBAERO laminar convective heating and radiative heating predictions with DPLR. As was evident in the centerline plots, CBAERO tends to under predict the laminar convective heating near the shoulder and over predict across the heat shield. For the radiative heating, CBAERO captures the correct maximum value but places the peak closer to the shoulder than does NEQAIR. Over the heat shield, CBAERO tends to slightly under predict the radiative heating.

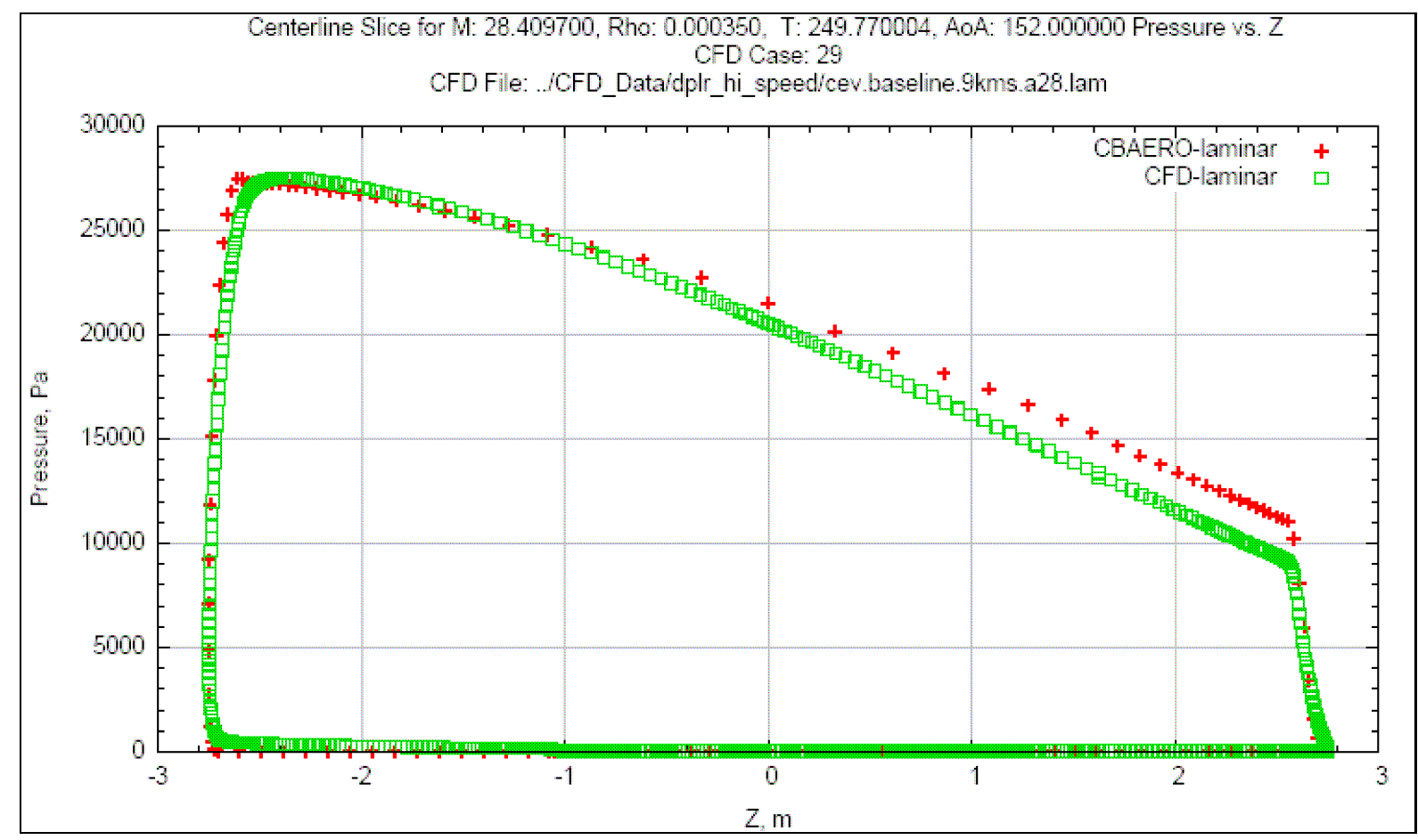

Fig. 4 Mach 28.4, 152.0 ${ }^{\circ}$ Angle of Attack, Centerline Pressure Distribution 


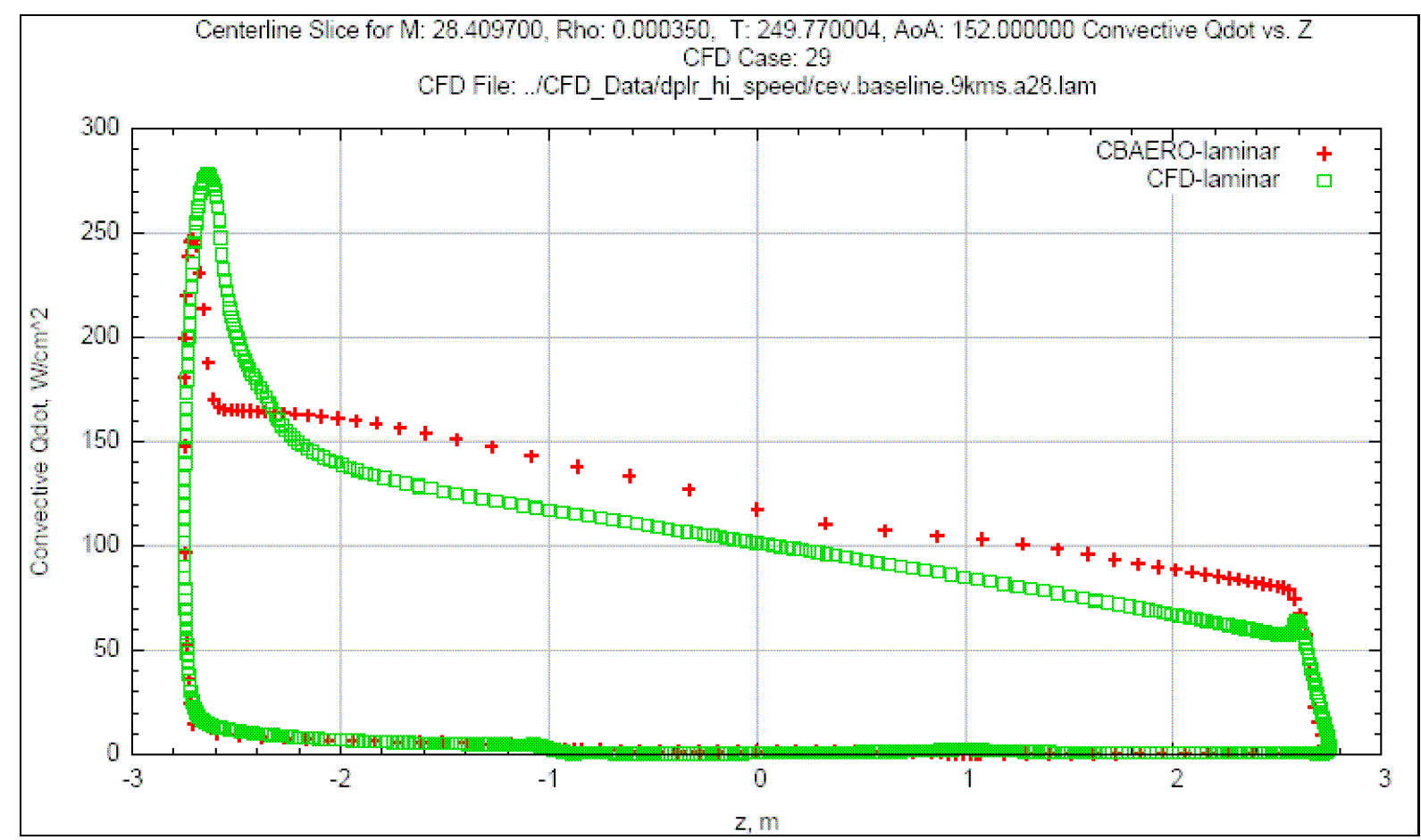

Fig. 5 Mach 28.4, 152.0 ${ }^{\circ}$ Angle of Attack, Centerline Laminar Convective Heating Distribution

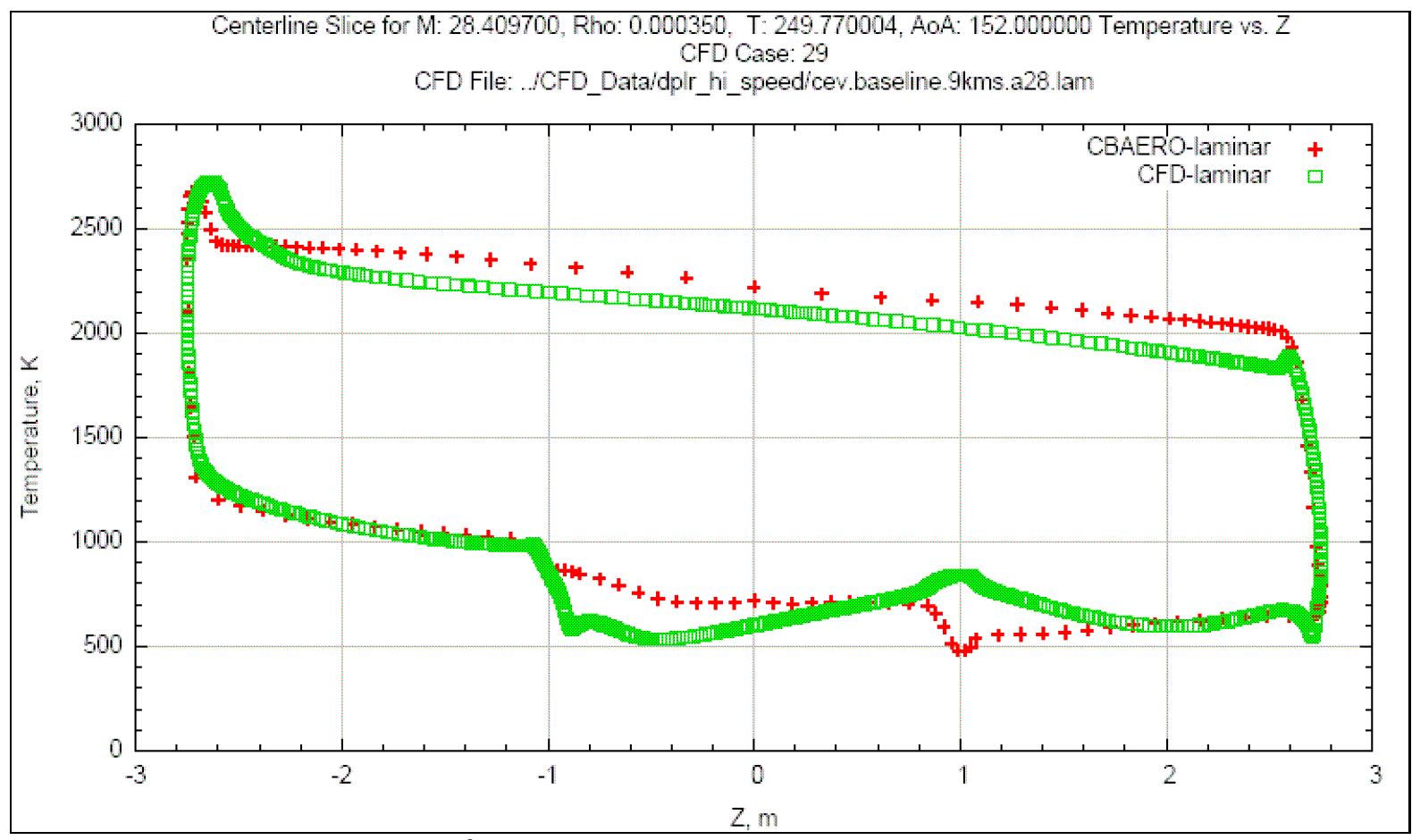

Fig. 6 Mach 28.4, 152.0 ${ }^{\circ}$ Angle of Attack, Centerline Laminar Temperature Distribution 


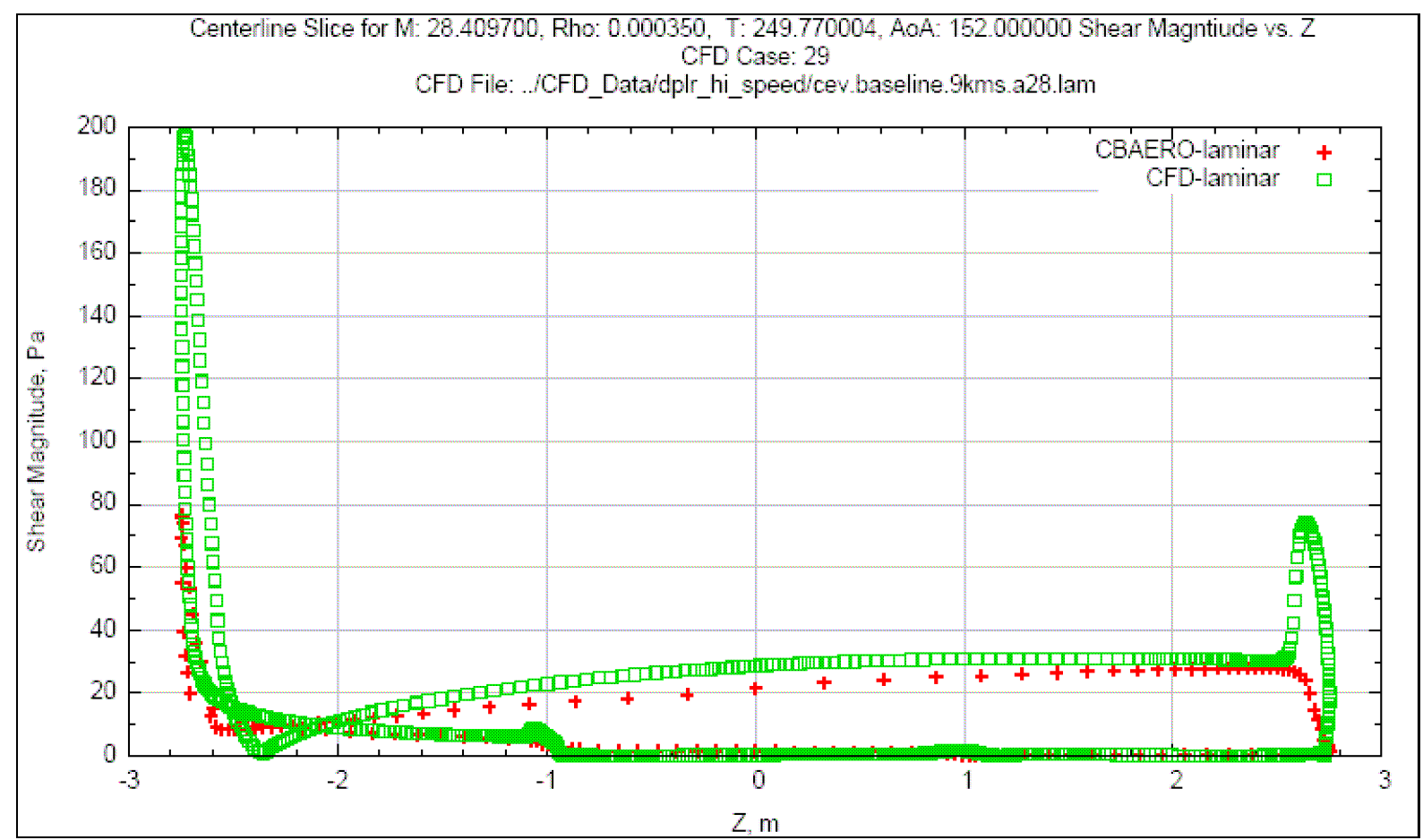

Fig. 7 Mach 28.4, 152.0 ${ }^{\circ}$ Angle of Attack, Centerline Laminar Shear Stress Magnitude Distribution

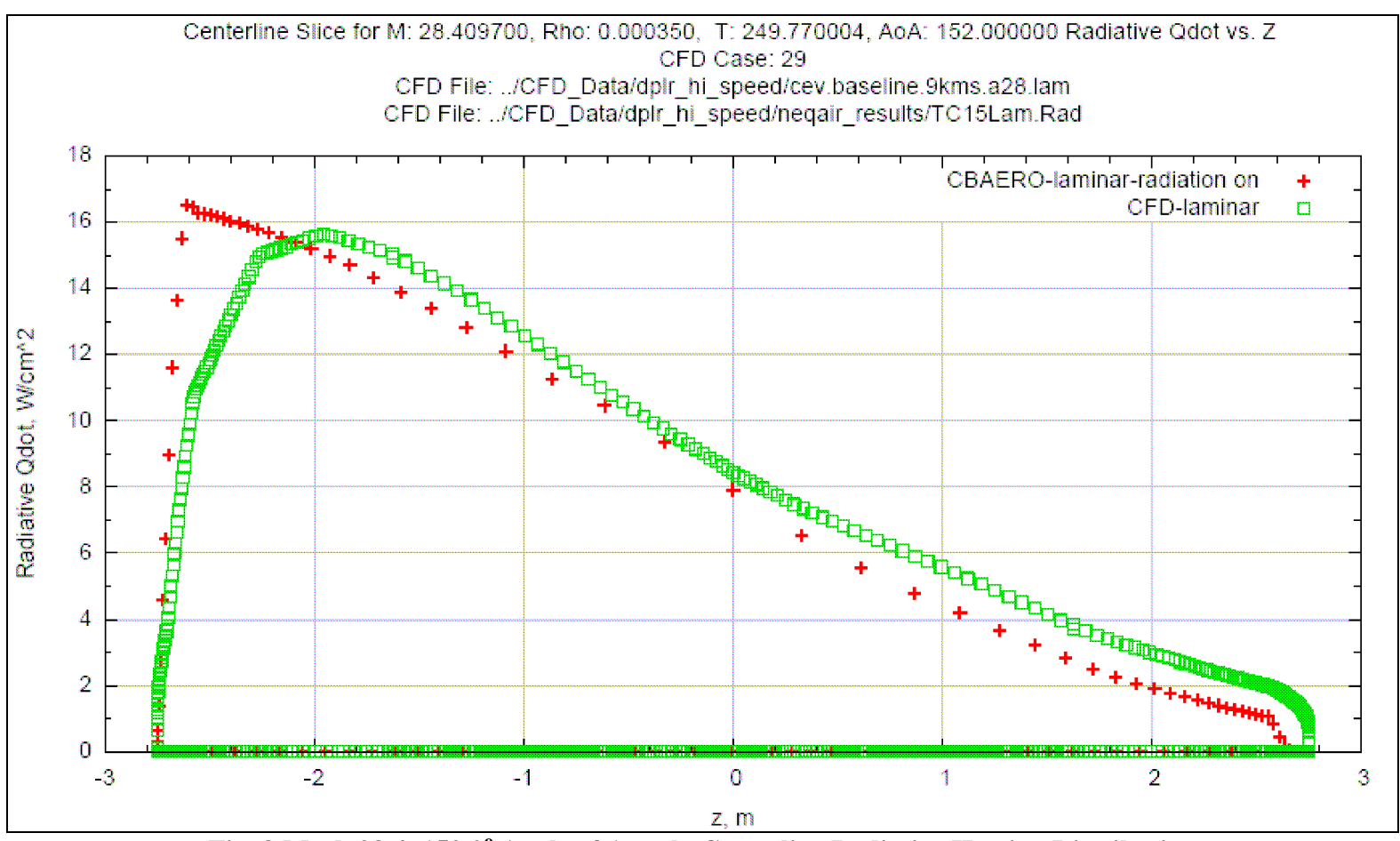

Fig. 8 Mach 28.4, 152.0 Angle of Attack, Centerline Radiative Heating Distribution 


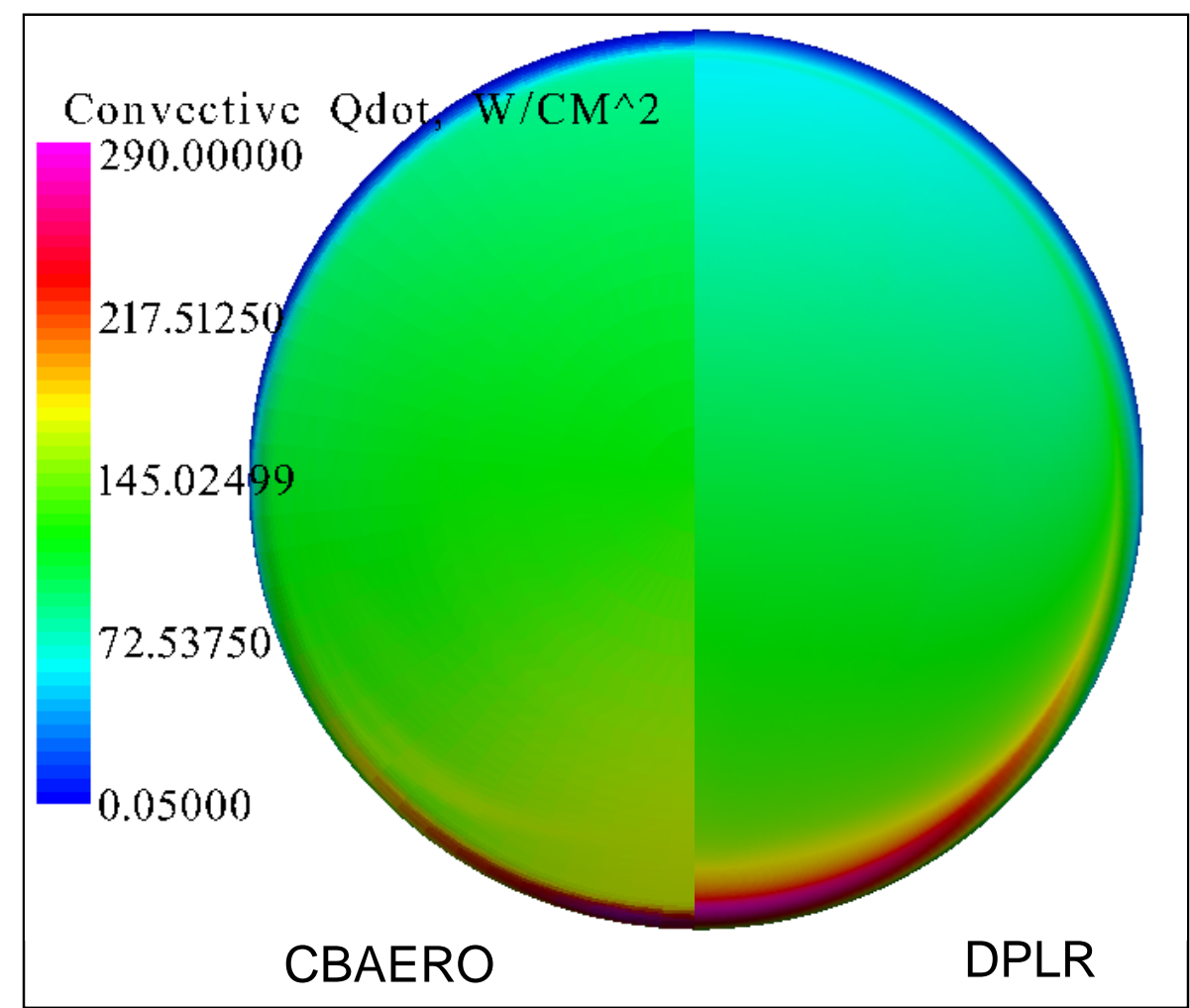

Fig. 9 Mach 28.4, 152.0 ${ }^{\circ}$ Angle of Attack, Surface Laminar Convective Heating - CBAERO solution compared with DPLR results.

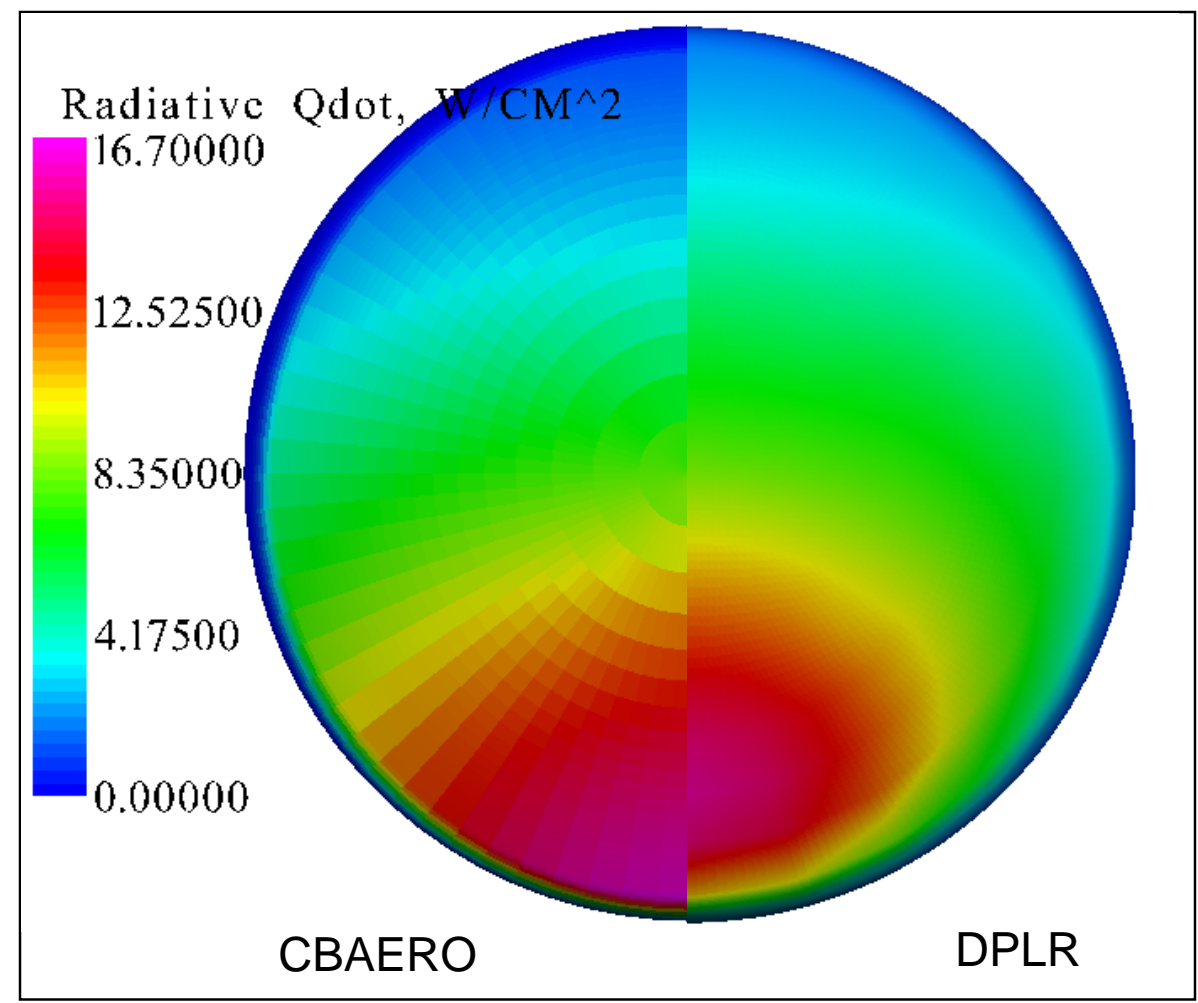

Fig. 10 Mach 28.4, 152.0 $^{\circ}$ Angle of Attack, Surface Radiative Heating - CBAERO solution compared with DPLR results. 


\section{The Anchoring Process}

The CPU requirements for CBAERO are minimal, typically requiring less than 10 seconds per solution on the current configuration and mesh. This is significantly less than that required for a single CFD solution from either DPLR [10] or LAURA $[15,16]$. To maximize the return on the computational resources invested in the DPLR and LAURA computations, the CFD solutions are used to anchor the CBAERO results.

At the limited number of free stream conditions (Mach, Dynamic Pressure, and Angle of Attack) at which CFD solutions are available the differences in the CFD and CBAERO solutions are compared. A companion piece of software is used to generate correction factors for the following CBAERO quantities: 1) pressure, 2) laminar convective heating, 3) turbulent convective heating, 4) laminar skin friction magnitude, 5) turbulent skin friction magnitude, 6) radiative heating, and 7) the boundary layer edge velocity components. The correction factors are calculated for each surface triangle for each CFD Mach-Q-Alpha condition. The corrections factors are then linearly interpolated in Mach-Q-Alpha, Mach-Q, Mach-Alpha, or Q-Alpha space as defined by the user. The linearly interpolated corrections are then used to anchor CBAERO solutions at free stream conditions for which no CFD solution is available.

\section{A. Pressure}

For the pressure corrections, the assumption is made that the laminar and turbulent pressure distributions are similar enough that only an anchoring using the laminar CFD solution is required. The pressure ratio correction is of the form

$$
\mathrm{P}_{\text {ratio }}=\frac{\text { CFD Pressure }}{\text { CBAEROPressure }}
$$

Here the pressures are the local pressures for each CBAERO surface triangle, obtained by interpolation from the original CFD surface mesh. The value of $\mathrm{P}_{\text {ratio }}$ is limited to values greater than 0 and less than 20.

\section{B. Convective Heating}

For the laminar and convective heating the correction ratios are of the form

$$
\begin{aligned}
\mathrm{C}_{\text {lam }_{\text {ratio }}}=\frac{\text { CFD Laminar } \dot{Q}}{\text { CBAEROLaminar } \dot{Q}} \\
\mathrm{C}_{\text {trb }_{\text {ratio }}}=\frac{\text { CFDTurbulent } \dot{Q}}{\text { CBAEROTurbulent } \dot{Q}}
\end{aligned}
$$

Here $\mathrm{Clam}_{\text {ratio }}$ and $\mathrm{Ctrb}_{\text {ratio }}$ refer to the correction ratios applied to the CBAERO estimates for the laminar and turbulent heat transfer coefficients. The values of the laminar and turbulent heating correction ratios are limited to values greater than zero and less than 10. Solving for $\mathrm{Clam}_{\text {ratio }}$ and $\mathrm{Ctrb}_{\text {ratio }}$ is an iterative process due to the nonlinear nature of the surface energy balance equation.

\section{Shear Forces}

The anchoring of the shear force magnitude requires a minor modification to the ratio formulation. Since shear force may go to zero, the correction ratio takes the form

$$
\mathrm{Cf}_{\text {lam }_{\text {rati }}}=\frac{\text { CFDShear Magnitude }}{\text { CBAEROShear Magnitude }+\varepsilon}
$$




$$
\mathrm{Cf}_{\text {trb }_{\text {ratio }}}=\frac{\text { CFDShear Magnitude }}{\text { CBAEROShear Magnitude }+\mathcal{E}}
$$

Here $\varepsilon$ is a small number, taken to be 0.25 Pascals. The values of $\mathrm{Cf}_{\text {lam }}$ ratio or $\mathrm{Cf}_{\text {trb }}$ ratio are limited to values greater than 0 and less than 10 .

\section{Radiative Heating}

The anchoring for radiative heating is similar to that for the shear magnitude

$$
\mathrm{Qs}_{\text {ratio }}=\frac{\text { CFD Radiative } \dot{Q}}{\text { CBAERO Radiative } \dot{Q}+\varepsilon}
$$

Once again, $\varepsilon$ is a small number, taken to be $0.25 \mathrm{Watts} / \mathrm{m}^{\wedge} 2$. The value of $\mathrm{Qs}_{\text {ratio }}$ is limited to values greater than 0 and less than 10 .

\section{E. Surface Streamlines}

The correction ratios calculated above for the laminar and turbulent skin friction account only for differences in the magnitude of the CBAERO and DPLR results. To further improve the CBAERO model, the differences in the CBAERO predicted surface streamline pattern, and those calculated by DPLR are accounted for. The CBAERO approximation for the local surface (or edge) velocity is simply

$$
\vec{V}_{\text {surface }}=\hat{n} \times \vec{V}_{\infty} \times \hat{n}
$$

Based on the $\mathrm{x}, \mathrm{y}$, and $\mathrm{z}$ components of the shear forces calculated by DPLR a correction factor can be calculated such that the adjusted surface (edge) velocity used by CBAERO accurately recovers the DPLR streamlines (based on shear). Unlike the previous correction ratios, the surface velocity corrections are deltas.

\section{F. Interpolating the Corrections to New Flight Conditions}

The correction ratios for pressure, convective heating, shear force magnitude, and radiative heating are calculated at each available CFD solution. The CFD anchoring 'space' is defined by a small number of CFD solutions in Mach - Dynamic Pressure - Alpha space. To apply these corrective ratios at any Mach-Q-Alpha condition an interpolation process must be defined. Currently, the CFD points are meshed in two or three dimensions using an unstructured tessellation of triangles or tetrahedra. The two or three dimensional unstructured mesh is created to satisfy the Delaunay criterion and thus enclose the convex hull of the CFD points. Figure 11 shows a hypothetical mesh in Mach-Q-Alpha space defined by a single tetrahedron. A similarly hypothetical and simple mesh in two dimensions would contain three nodes and a single triangle.

Linear interpolation is performed on the unstructured mesh to calculate the correction ratios for Mach-Q-Alpha condition that lies within the convex hull of the CFD points. For a Mach-Q-Alpha condition outside the convex hull value returned from the interpolation (extrapolation) is 'clipped' to the edge of the 'table'. Hence extrapolation of the corrections is explicitly forbidden. 


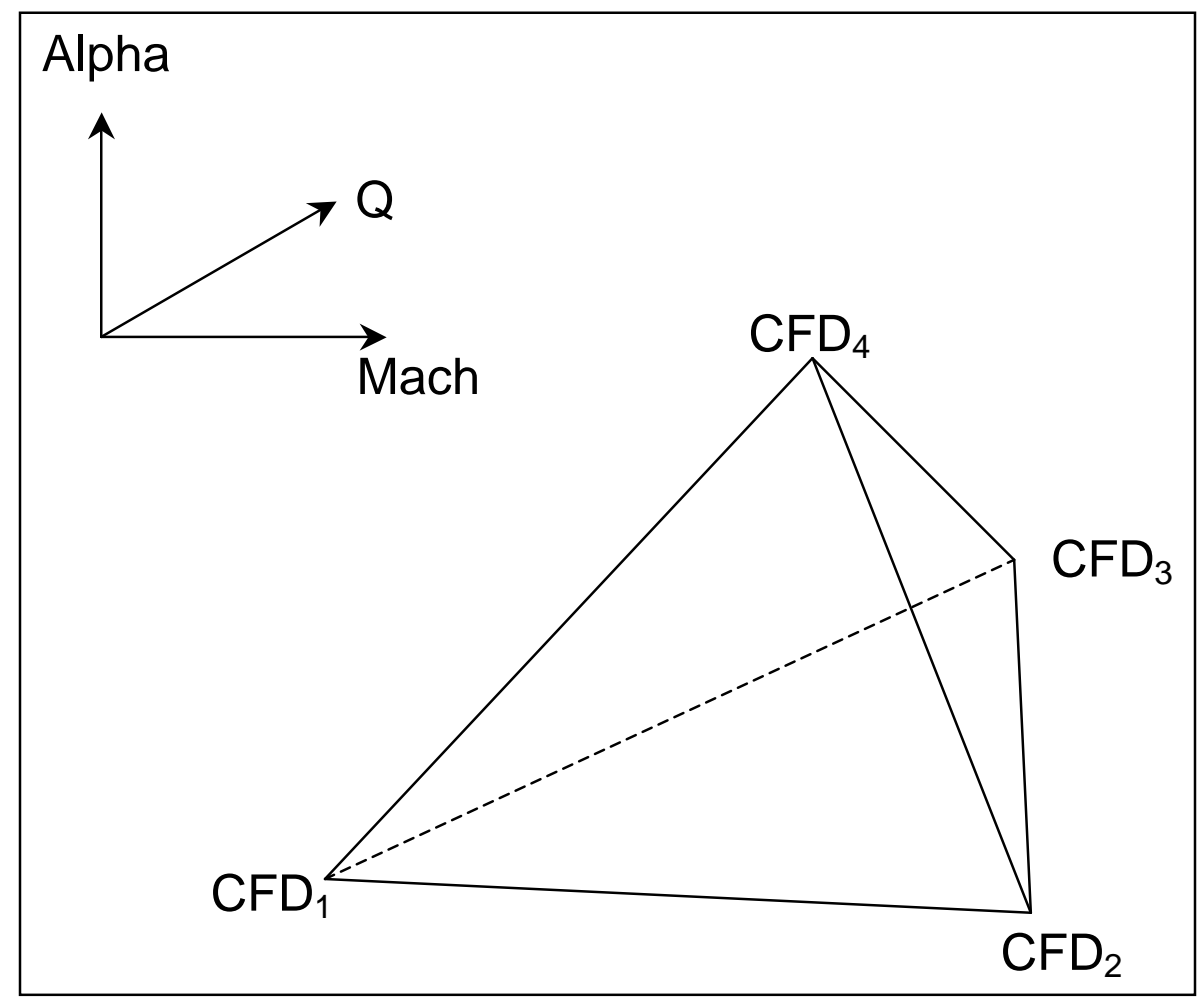

Fig. 11 Hypothetical CFD anchoring mesh comprised of a single tetrahedron in Mach-Q-Alpha space.

\section{Testing of the Process}

As a test, of the anchoring procedure, approximately $1 / 2$ of the CFD solutions available for anchoring are removed from the 'anchoring list'. The remaining CFD solutions are then used to create the anchoring database as described above. CBAERO is then run, using the anchoring corrections, at the reserved CFD conditions and comparisons are made. The total list of available CFD solutions is presented in Table 1. The first 14 CFD solutions in Table 1, highlighted in red, are used in the anchoring procedure. These first 14 CFD solutions are those originally generated during NASA's Exploration Systems Architecture Study (ESAS). Figures 12 and 13 present the MachAlpha tessellation of the CFD point set used for this anchoring test.

Figure 12 shows the two dimensional tessellation used to interpolate the correction ratios in Mach-Alpha space. Figure 13 shows the true distribution of the CFD anchor points in the complete three dimensional Mach-Alpha-Q space in which they reside. The uneven and sparse nature of the dynamic pressure variance $(\mathrm{Q})$ of the CFD anchor points was the driver for restricting the interpolation to two dimensions (Mach and Alpha).

Figures 14 through 18 present the anchored CBAERO results, using the first 14 CFD solutions, compared to DPLR for the Mach $28.4,152^{\circ}$ case presented earlier. These results should be compared with figures 4 through 8 .

The anchored CBAERO pressure distribution along the centerline, shown in figure 14, matches the DPLR results very nicely. Some differences are noticeable on the leeward side, where the anchoring ratios were likely clipped. Figure 15 compares the anchored CBAERO results for the laminar convective heating with DPLR. Once again, the anchored results compare very nicely with the exact DPLR results, even on the leeward side. Figure 16 compares the anchored CBAERO temperature distribution, highlighting the leeward heating environment. Once again, the anchored CBAERO results compare very favorably with the exact DPLR solution. Figure 17 presents the anchored CBAERO laminar shear magnitude results. The anchored results miss the peak shear magnitude on the shoulder by approximately $20 \%$, however this is an improvement over the unanchored results which missed the shear magnitude by a factor of 2.5. Finally, figure 18 compares the anchored CBAERO radiative heating prediction with 
the NEQAIR results. The anchored CBAERO more closely matches the NEQAIR radiative heating result in both magnitude and general shape.

Figures 19 and 20 compare the surface distribution on the anchored CBAERO laminar convective and radiative heating with the DPLR and NEQAIR results. These anchored CBAERO results can be compared with those in figures 9 and 10.

\section{Final Aerothermal Database Creation}

The final anchored CBAERO laminar and turbulent databases use all of the available CFD anchor points from DPLR, LAURA, and NEQAIR. Figure 21 shows the two dimensional anchoring mesh used in Mach-Alpha space. Figure 22 shows the true distribution of the anchoring points in the complete Mach-Alpha-Q space. Once again, due to the uneven and sparse nature of the CFD anchor points in the Q-dimension the interpolation of the anchoring ratios was limited to Mach-Alpha space.

Table 2 lists the Mach, Q, and Alpha conditions covered in the anchored laminar and turbulent databases. The anchored databases include 25 Mach numbers, 19 dynamic pressures, and 11 angles of attack for a total of 5225 cases. Figures 23 and 24 show the distribution of the database points with the CFD anchoring points overlaid. Both databases were run on NASA's Columbia super computer using 55 processors. The anchored laminar database required 10 wall clock minutes, while the anchored turbulent database required 15 wall clock minutes. These timings average to approximately 6 to 10 seconds per solution.

Figures 25 through 31 compare the anchored CBAERO solution with the DPLR and NEQAIR results. Since this Mach 28.4 $152^{\circ}$ case is now part of the anchoring CFD cases the results shown in these figures should, within the limitations imposed by the ratio limiters, recover the CFD solution. This is true for all the quantities for which anchoring is applied. The largest discrepancies appear in the maximum shear force near the shoulder, and near the point of minimum radiative heating on the leeward shoulder.

\section{Conclusions and Future Work}

The CBAERO software package has been used to predict the convective and radiative heating environments for the Crew Exploration Vehicle (CEV). A limited number of high fidelity CFD solutions are used to 'anchor' the engineering level estimates of pressure, convective heating, radiative heating, and shear stress. This mixture of fast engineering level analysis, anchored by high fidelity CFD, provides for a very flexible tool during conceptual and preliminary design. The used of CBAERO and the anchoring process increases the value of the available CFD solutions beyond the single flight condition at which they were original run. The anchoring processed described here is currently being used to support the CEV program, and on going work is aimed at extending the anchoring process to include additional boundary layer edge information extracted from the CFD solutions, as well as extending the process to include rarefied flow solutions from NASA's Direct Simulation Monte Carlo (DSMC) code DAC [20].

\section{References}

[1] Kinney, D.J., "Aero-Thermodynamics for Conceptual Design”, AIAA Paper 13382, January 2004, Reno Nevada

[2] Kenwright, David N., "Feature Extraction of Separation and Attachment Lines", IEEE Transactions on Visualization and Computer Graphics, Vol. 5, No. 2, April-June 1999

[3] Barnes, J.E., "N-Body Models of Collisionless Systems", volume 1, chapter 8, Springer-Verlag, 1996

[4] Barnes, J.E., and Hut, P., "A hierarchical O(N Log N) force calculation algorithm", Nature, 324(4):446-449, December 1986

[5] Tauber, Michael E., "A Review of High-Speed Convective, Heat-Transfer Computation Methods", NASA TP 2914,1989

[6] Goulard, R., "Recombination Rates in Hypersonic Stagnation Heat Transfer", in An Introduction to Hypersonic Aerodynamics, presented at the ARS Annual Meeting, NY, December, 1957.

[7] Stewart, David A., "Surface Catalysis and Characterization of Proposed Candidate TPS for Access-to-Space Vehicles", NASA Technical Memorandum, 112206, July 1997.

[8] McBride, B. J. and Gordon, S., "Computer Program for Calculation of Complex Chemical Equilibrium Compositions and Applications II. User's Manual and Program Description", NASA RP-1311-P2, June 1996

[9] Tauber, M.E. and Sutton, K., "Stagnation-Point Radiative Heating Relations for Earth and Mars Entries", Journal of Spacecraft Volume 28, Number 1, January-February 1991, Pages 40-42 
[10] Wright, M.J., Prabhu, D.K., and Martinez, E.R., "Analysis of Afterbody Heating Rates on the Apollo Command Modules, Part1: AS-202", AIAA Paper 2004-2456, 37th AIAA Thermophysics Conferenence

[11] Whiting, E.E., Yen, L., Arnold, J.O., and Paterson, J.A, "NEQAIR96", Nonequilibrium and Equilibrium Radiative Transport and Spectra Program: User's Manual", NASA RP-1389, Dec. 1996

[12] Lee, D.B. and Goodrich, W.D., "Heat Transfer Rate and Prssure Measurements Obtained during Apollo Orbital Entries", NASA TN D=6028, Oct. 1970

[13] Cornette, E.S., "Forebody Temperatures and Calorimeter Rates Measured During Project Fire II Reentry at $11.35 \mathrm{~km} / \mathrm{s} "$, NASA TM X-1305, Nov. 1966

[14] Adelman, H. "HAVOC Aerothermal Heating (Partially Catalytic Surfaces)". ELORET Task 8, Contract No. NAS2-0062, Report No 2, February 2001.

[15] Gnoffo, P. A., Gupta, R. N., and Shinn, J., "Conservation Equations and Physical Models for Hypersonic Air Flows in Thermal and Chemical Nonequilibrium," NASA TP 2867, 1989

[16] Gnoffo, P. A., "An Upwind-Biased, Point-Implicit Relaxation Algorithm for Viscous, Compressible PerfectGas Flows," NASA TP 2953, 1990

[17] Zoby, E.V, Moss, J.N., and Sutton, K. "Approximate Convective-Heating Equations for Hypersonic Flows", Journal of Spacecraft and Rockets, Vol. 18: pp. 64-70, January-February 1981.

[18] Bird, G.A., Molecular Gas Dynamics and the Direct Simulation of Gas Flows, Clarendon Press, Oxford 1994.

[19] Wilmoth, R.G. and Blanchard, R.C. "Rarefied Transitional Bridging of Blunt Body Aerodynamics"; 21st International Symposium on Rarefied Gas Dynamics

[20] LeBeau, G.J. "A Parallel Implementation of the Direct Simulation Monte Carlo Method," Computer Methods in Applied Mechanics and Engineering Parallel Computational Methods for Flow Simulation and Modeling, Vol 174, pp 319-337. AIAA Paper 2006-3577, June 2006. 


\begin{tabular}{|c|c|c|c|c|c|c|}
\hline Mach & Density & Temp & Alpha & Lam & Trb & Rad \\
\hline & $\mathrm{kg} / \mathrm{m}^{\wedge} 3$ & ${ }^{\circ} \mathrm{K}$ & deg & $y / n$ & $y / n$ & $y / n$ \\
\hline \multicolumn{7}{|c|}{ ESAS Study Runs } \\
\hline 6 & 0.0040000 & 250.00 & 147.00 & Yes & Yes & No \\
\hline 6 & 0.0040000 & 250.00 & 157.00 & Yes & Yes & No \\
\hline 10 & 0.0020000 & 250.00 & 147.00 & Yes & Yes & No \\
\hline 10 & 0.0020000 & 250.00 & 157.00 & Yes & Yes & No \\
\hline 18 & 0.0004000 & 250.00 & 147.00 & Yes & Yes & No \\
\hline 18 & 0.0004000 & 250.00 & 157.00 & Yes & Yes & No \\
\hline 21.44 & 0.0016271 & 267.97 & 153.00 & Yes & Yes & No \\
\hline 21.44 & 0.0016271 & 267.97 & 152.50 & Yes & Yes & No \\
\hline 25 & 0.0000400 & 250.00 & 147.00 & Yes & Yes & No \\
\hline 25 & 0.0000400 & 250.00 & 157.00 & Yes & Yes & No \\
\hline 28.93 & 0.0006269 & 263.08 & 153.00 & Yes & Yes & Yes \\
\hline 29.54 & 0.0005378 & 259.51 & 153.00 & Yes & Yes & Yes \\
\hline 32.2 & 0.0003396 & 248.89 & 147.00 & Yes & Yes & Yes \\
\hline 32.2 & 0.0003396 & 248.89 & 157.00 & Yes & Yes & Yes \\
\hline \multicolumn{7}{|c|}{ DPLR LEO Runs } \\
\hline 23.84 & 0.0001632 & 233.29 & 150.00 & Yes & Yes & No \\
\hline 23.84 & 0.0001632 & 233.29 & 152.00 & Yes & Yes & No \\
\hline 23.84 & 0.0001632 & 233.29 & 154.00 & Yes & Yes & No \\
\hline 23.621 & 0.0001412 & 230.30 & 150.00 & Yes & Yes & No \\
\hline 23.621 & 0.0001412 & 230.30 & 154.00 & Yes & Yes & No \\
\hline 16.78 & 0.0004073 & 253.15 & 150.00 & Yes & Yes & No \\
\hline 16.78 & 0.0004073 & 253.15 & 152.00 & Yes & Yes & No \\
\hline 16.78 & 0.0004073 & 253.15 & 154.00 & Yes & Yes & No \\
\hline 9.63 & 0.0064270 & 241.50 & 150.00 & Yes & Yes & No \\
\hline 23.82 & 0.0001412 & 230.30 & 152.84 & Yes & Yes & No \\
\hline \multicolumn{7}{|c|}{ LAURA LEO Runs } \\
\hline 9.26 & 0.0022590 & 261.40 & 150.00 & Yes & Yes & No \\
\hline 9.26 & 0.0022590 & 261.40 & 152.00 & Yes & Yes & No \\
\hline 9.26 & 0.0022590 & 261.40 & 154.00 & Yes & Yes & No \\
\hline 20.4 & 0.0005045 & 257.98 & 152.00 & Yes & Yes & No \\
\hline 23.82 & 0.0001412 & 230.30 & 152.84 & Yes & Yes & No \\
\hline \multicolumn{7}{|c|}{ DPLR High Speed Cases } \\
\hline 28.4097 & 0.0003505 & 249.77 & 152.00 & Yes & Yes & Yes \\
\hline 35.78605 & 0.0000624 & 214.26 & 152.00 & Yes & Yes & Yes \\
\hline
\end{tabular}

Table. 1 CFD anchoring points at which DPLR, LAURA, and/or NEQAIR solutions were available. The points in red indicate those CFD solutions used in the testing of the anchoring process. The LAURA solution in blue is a 'duplicate' of a DPLR solution. All CFD solutions are based on a $5.5 \mathrm{~m}$ diameter CEV. 


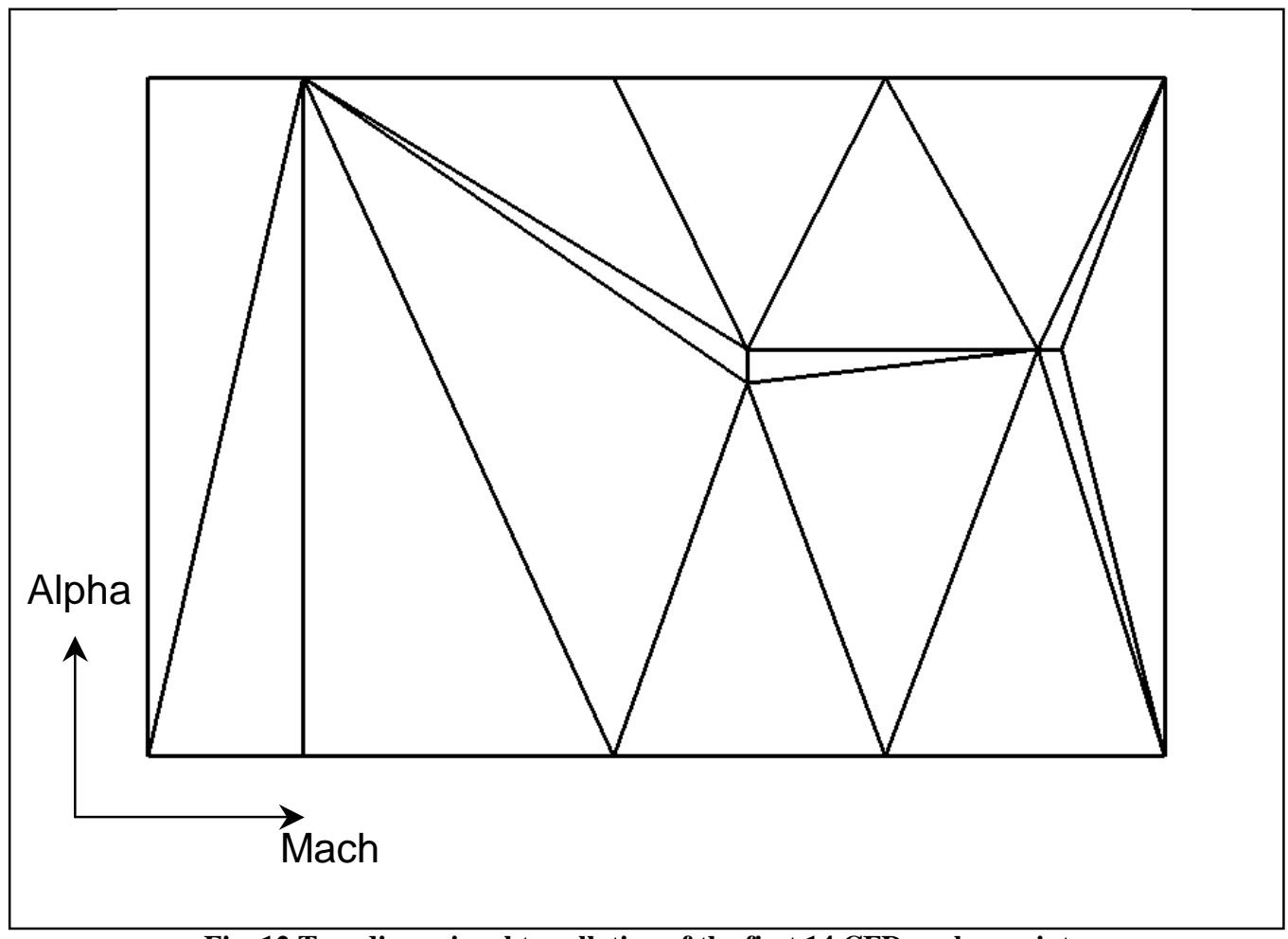

Fig. 12 Two dimensional tessellation of the first 14 CFD anchor points.

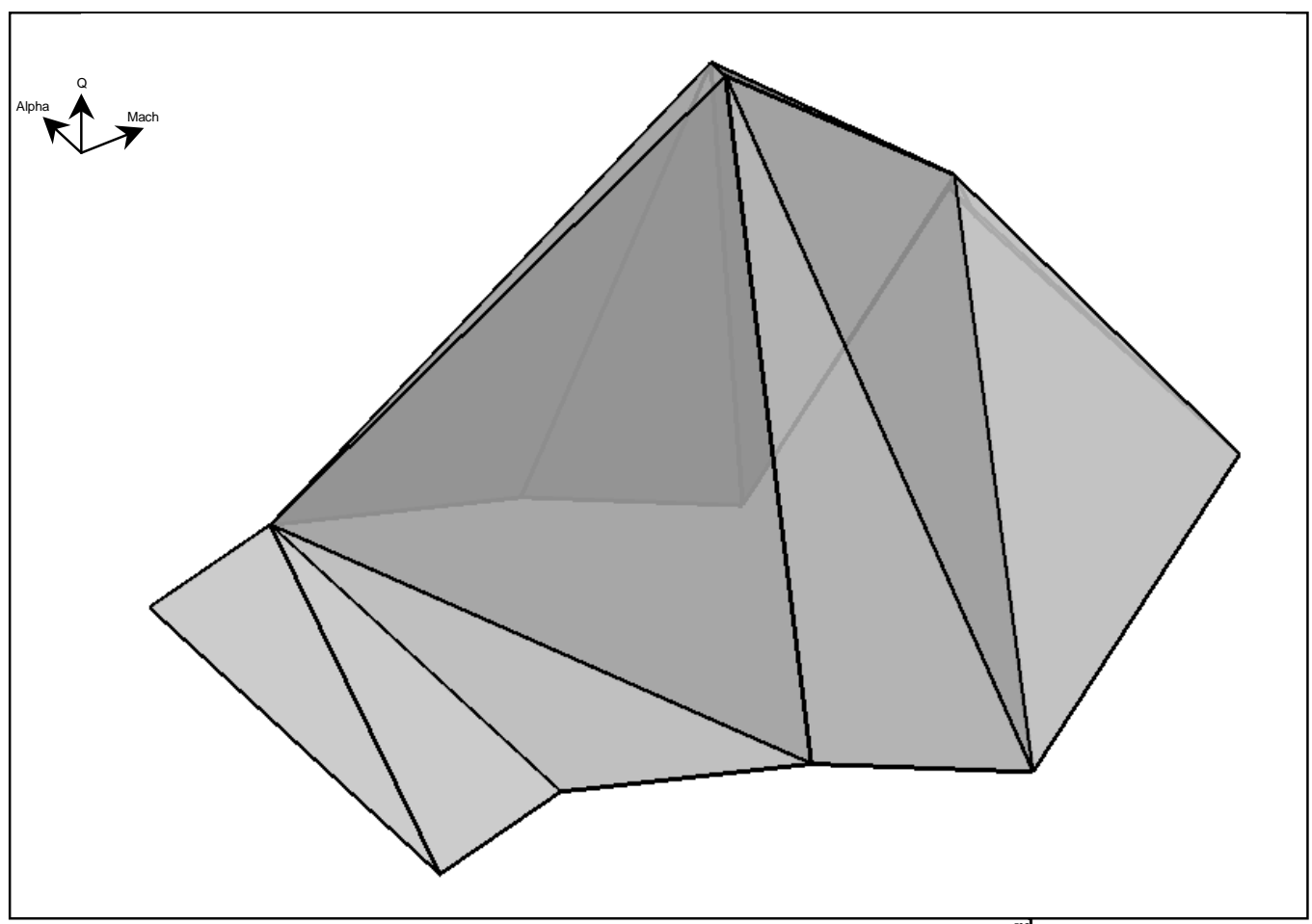

Fig. 13 "Two dimensional" tessellation of the first 14 CFD anchor points with the $3^{\text {rd }}$ dimension (dynamic pressure) shown. 


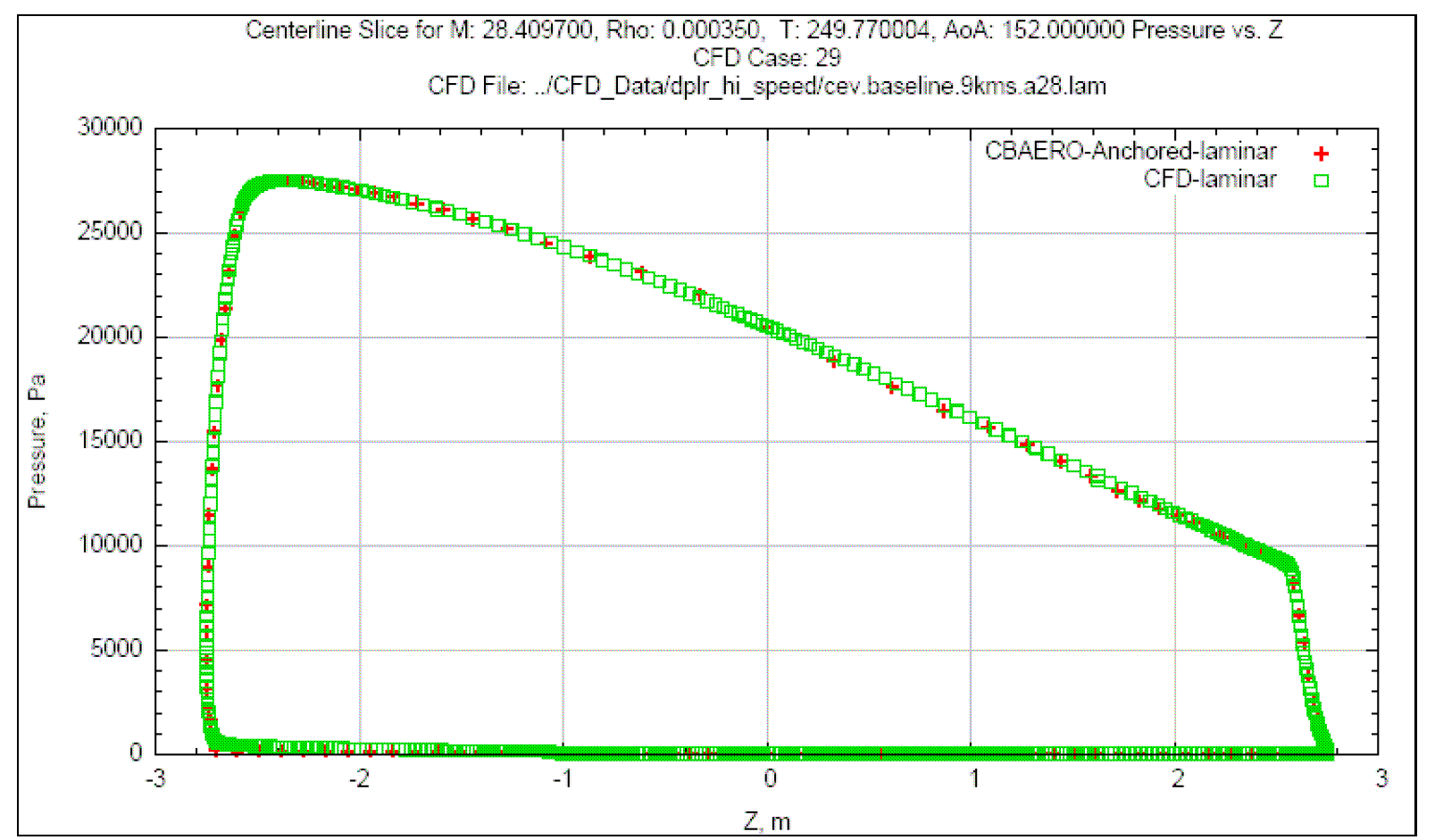

Fig. 14 Mach 28.4, 152.0 Angle of Attack, Centerline Pressure Distribution - Anchored CBAERO solution compared with DPLR results. Only $1 / 2$ of the available CFD points were used in the anchoring process, leaving the remaining CFD points, such as this one, for use as test cases.

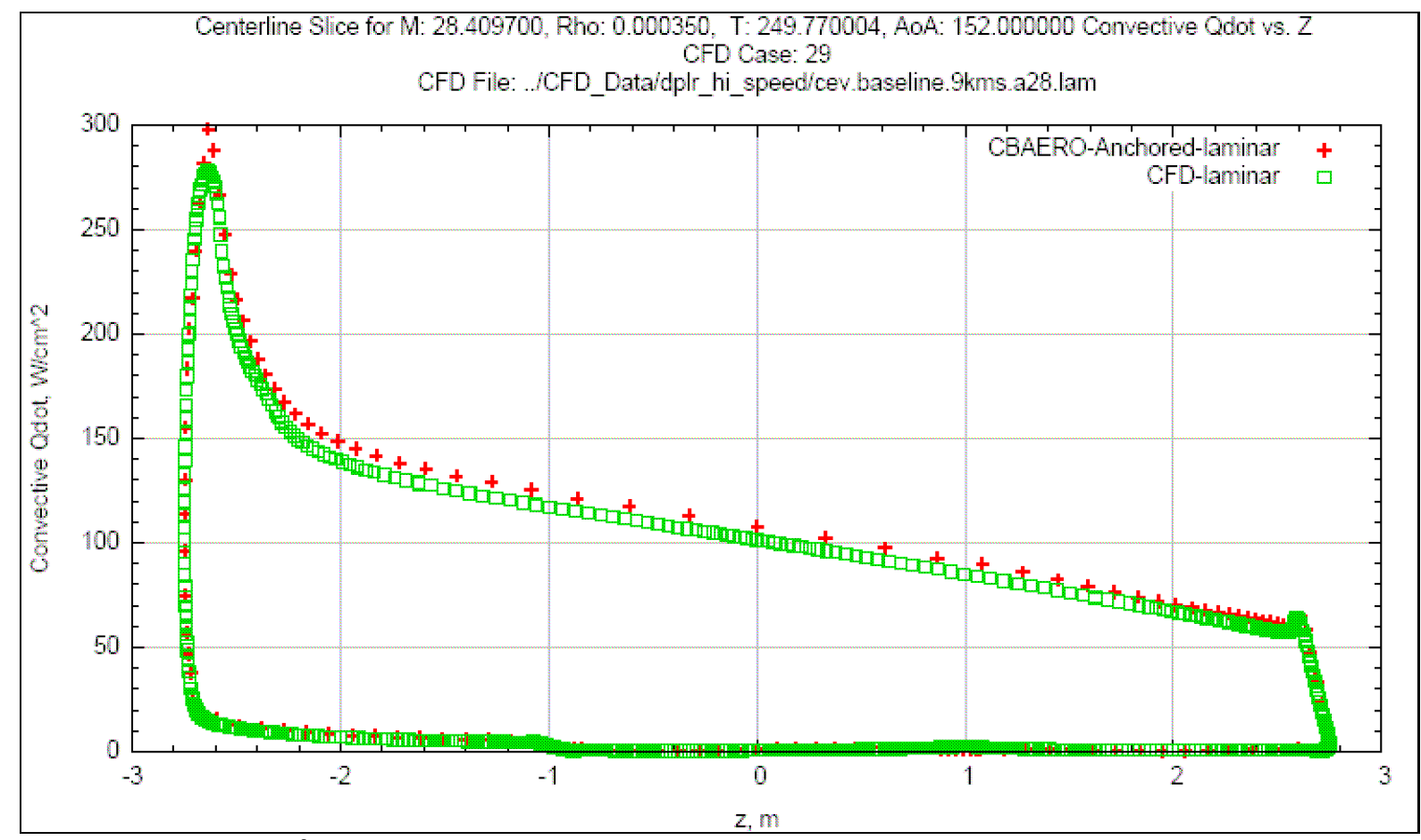

Fig. 15 Mach 28.4, 152.0 ${ }^{\circ}$ Angle of Attack, Centerline Laminar Convective Heating Distribution - Anchored CBAERO solution compared with DPLR results. Only $1 / 2$ of the available CFD points were used in the anchoring process, leaving the remaining CFD points, such as this one, for use as test cases. 


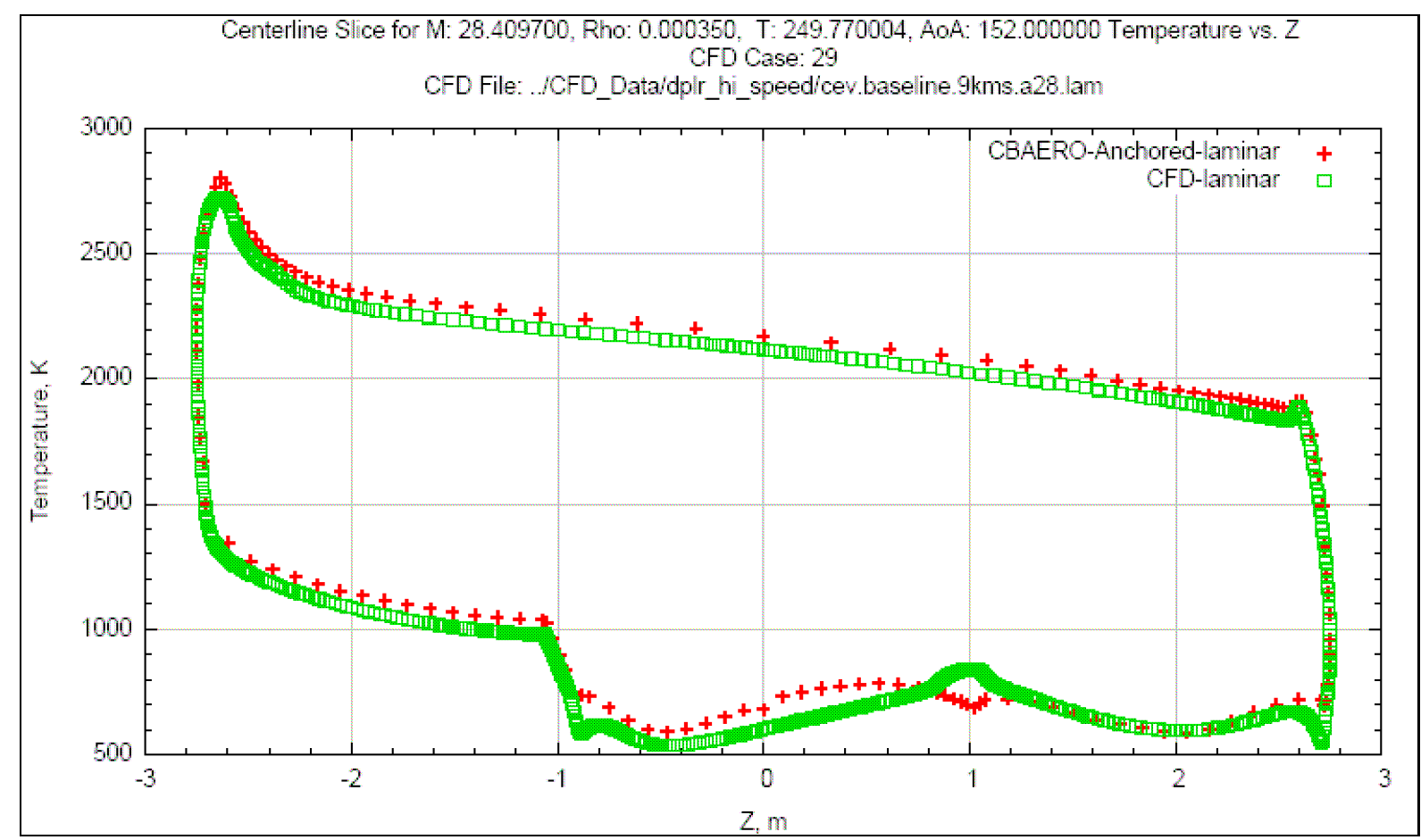

Fig. 16 Mach 28.4, 152.0 ${ }^{\circ}$ Angle of Attack, Laminar Temperature Distribution - Anchored CBAERO solution compared with DPLR results. Only $1 / 2$ of the available CFD points were used in the anchoring process, leaving the remaining CFD points, such as this one, for use as test cases.

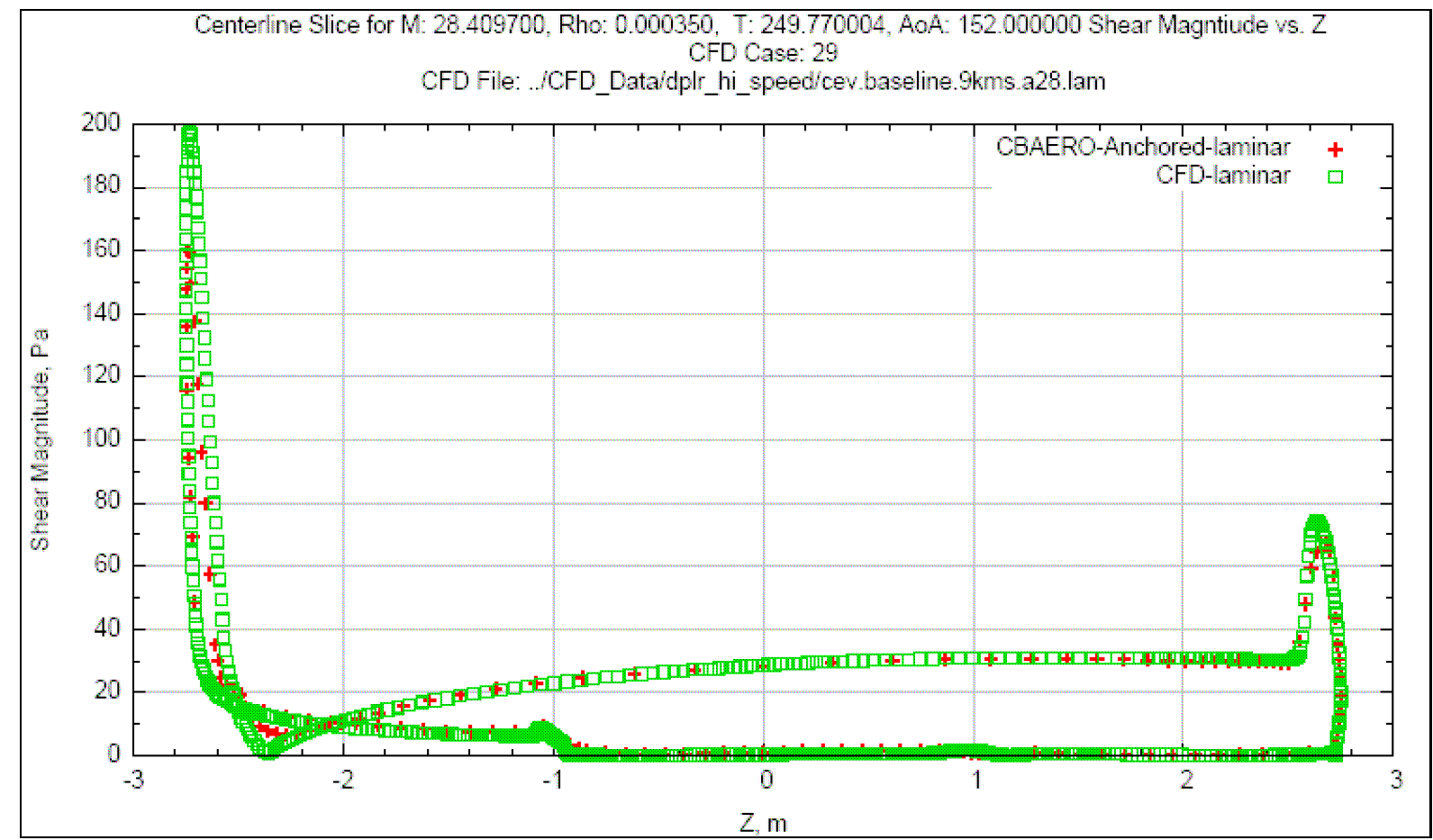

Fig. 17 Mach 28.4, 152.0 ${ }^{\circ}$ Angle of Attack, Centerline Laminar Shear Stress Magnitude - Anchored CBAERO solution compared with DPLR results. Only $1 / 2$ of the available CFD points were used in the anchoring process, leaving the remaining CFD points, such as this one, for use as test cases. 


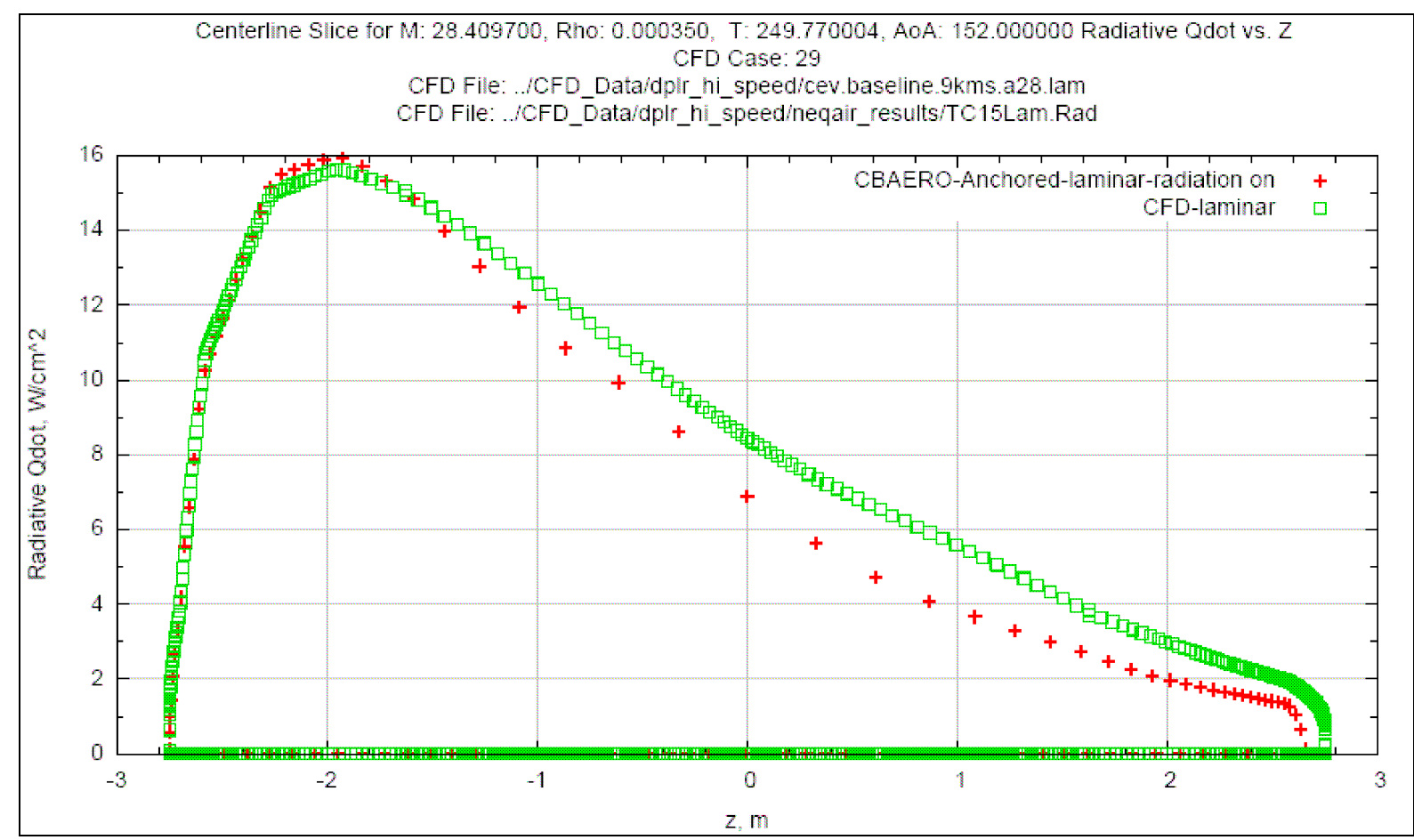

Fig. 18 Mach 28.4, $152.0^{\circ}$ Angle of Attack, Centerline Radiative Heating - Anchored CBAERO solution compared with NEQAIR results. Only $1 / 2$ of the available CFD points were used in the anchoring process, leaving the remaining CFD points, such as this one, for use as test cases.

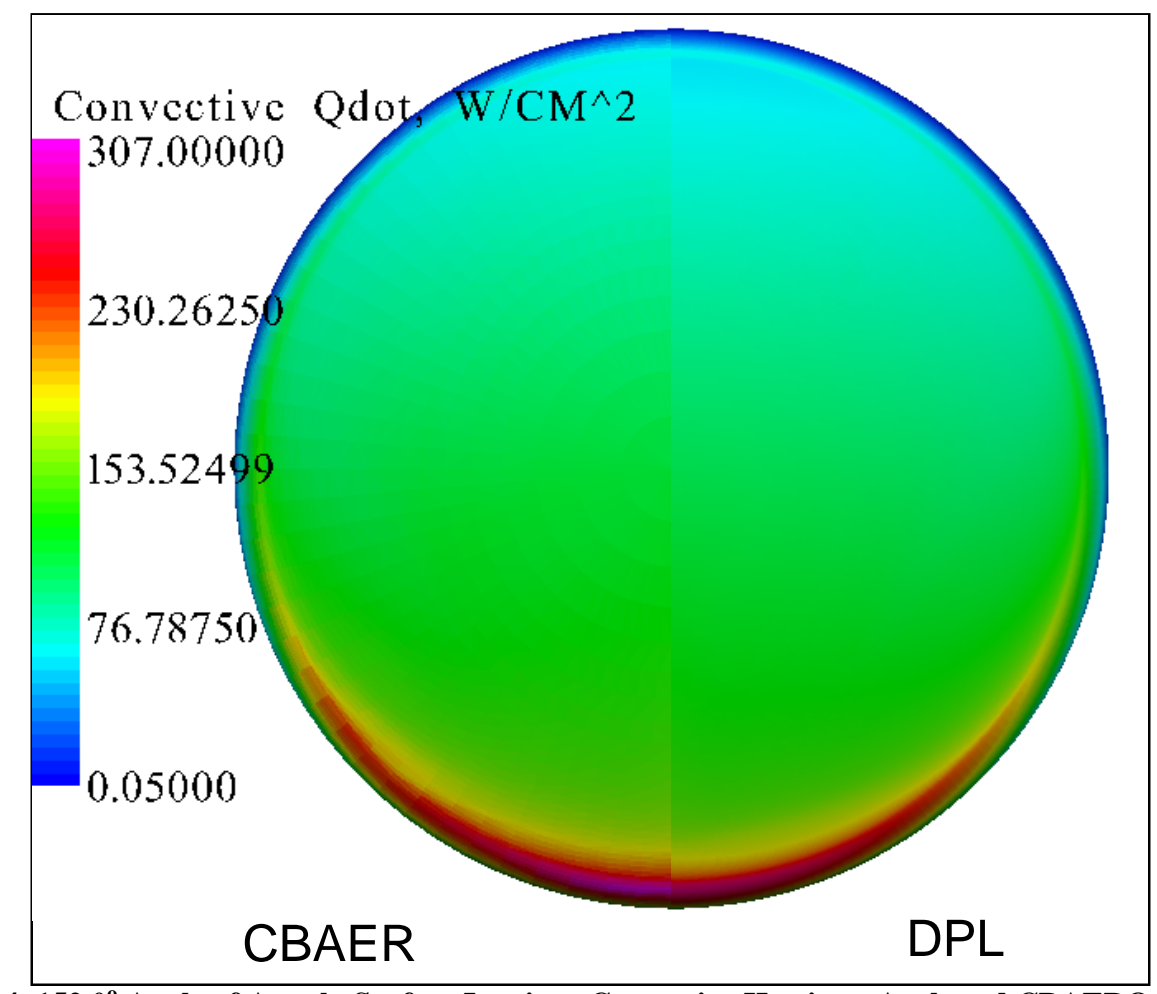

Fig. 19 Mach 28.4, 152.0 $^{\circ}$ Angle of Attack, Surface Laminar Convective Heating - Anchored CBAERO solution compared with DPLR results. Only $1 / 2$ of the available CFD points were used in the anchoring process, leaving the remaining CFD points, such as this one, for use as test cases 


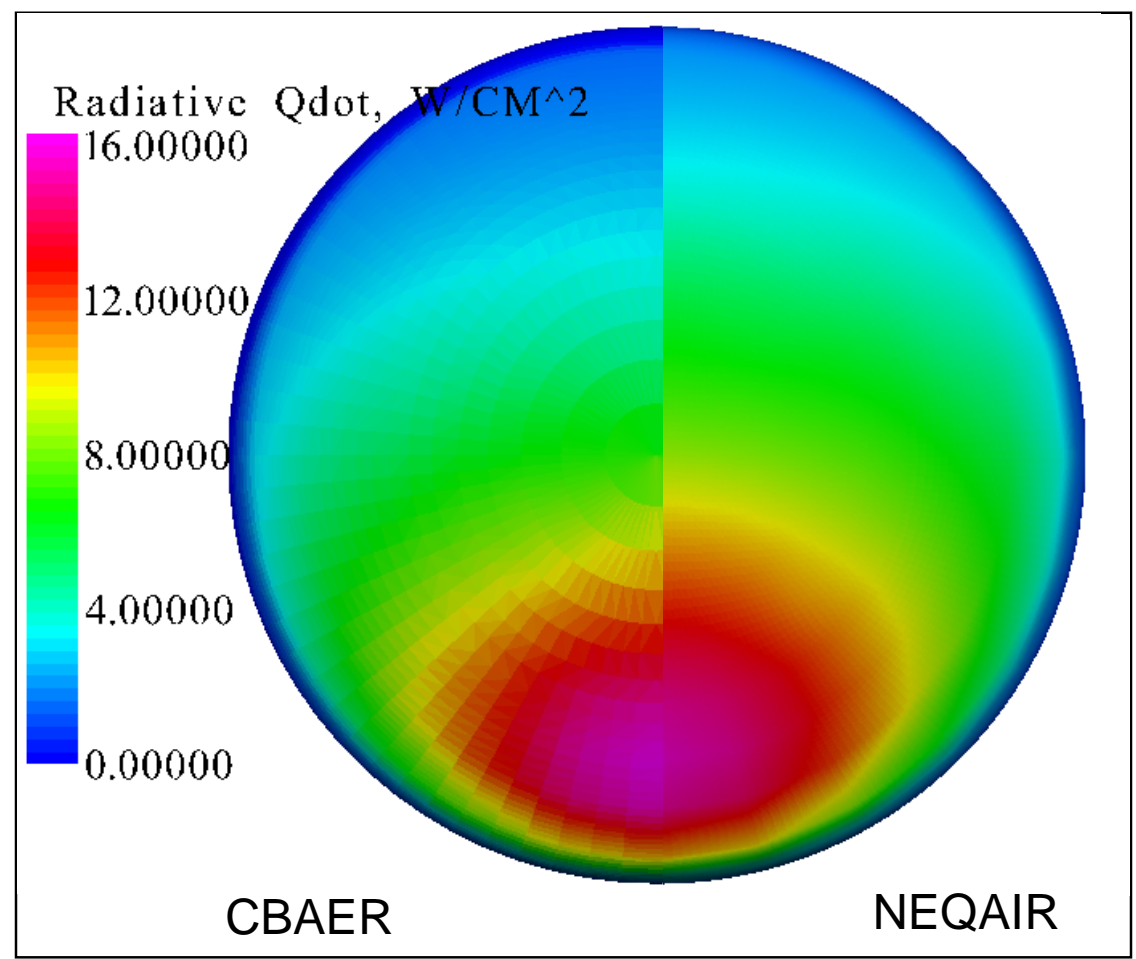

Fig. 20 Mach 28.4, 152.0 ${ }^{\circ}$ Angle of Attack, Surface Radiative Heating - Anchored CBAERO solution compared with DPLR results. Only $1 / 2$ of the available CFD points were used in the anchoring process, leaving the remaining CFD points, such as this one, for use as test cases.

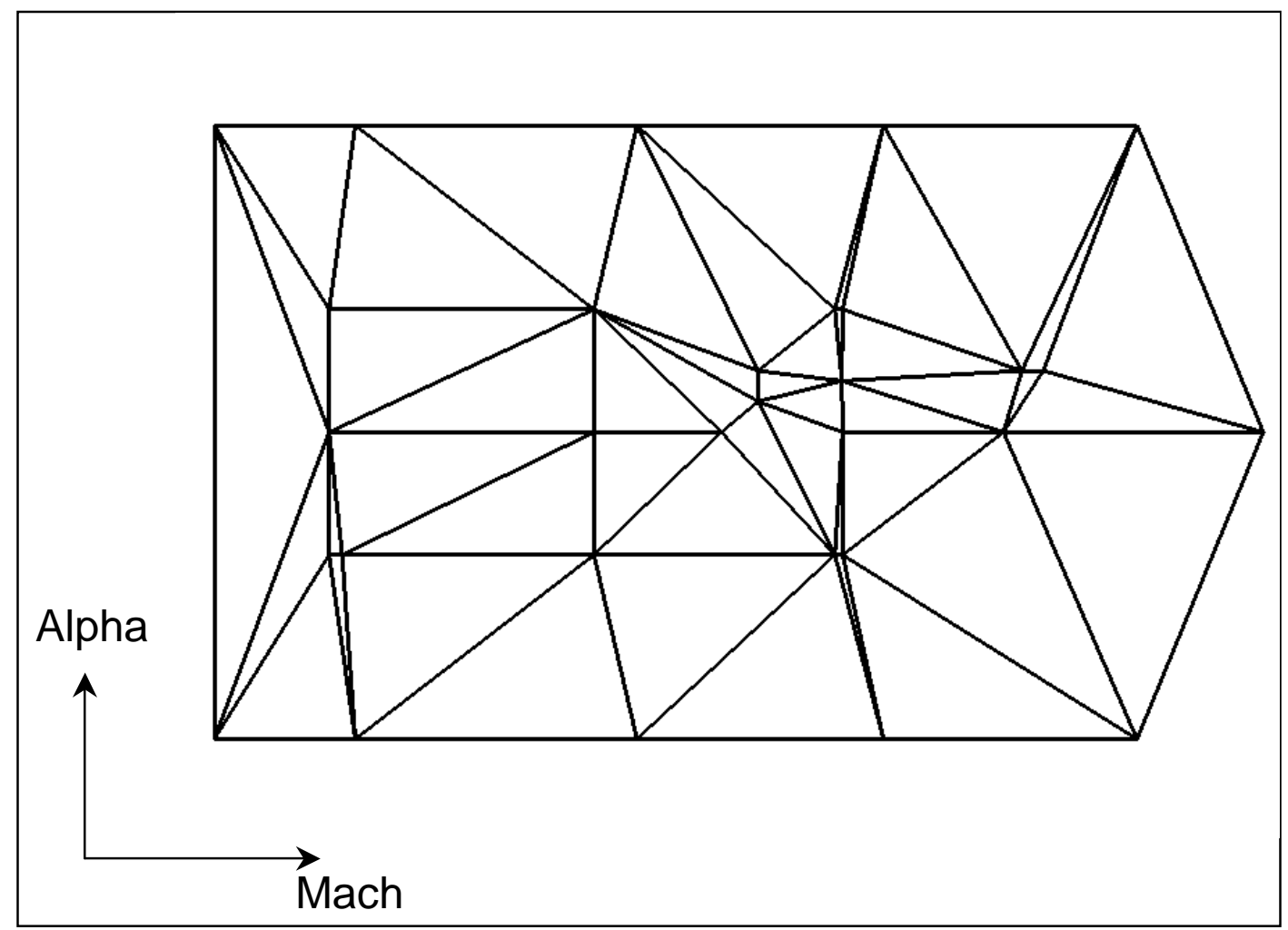

Fig. 21 Two dimensional tessellation of all the available CFD anchor points. 


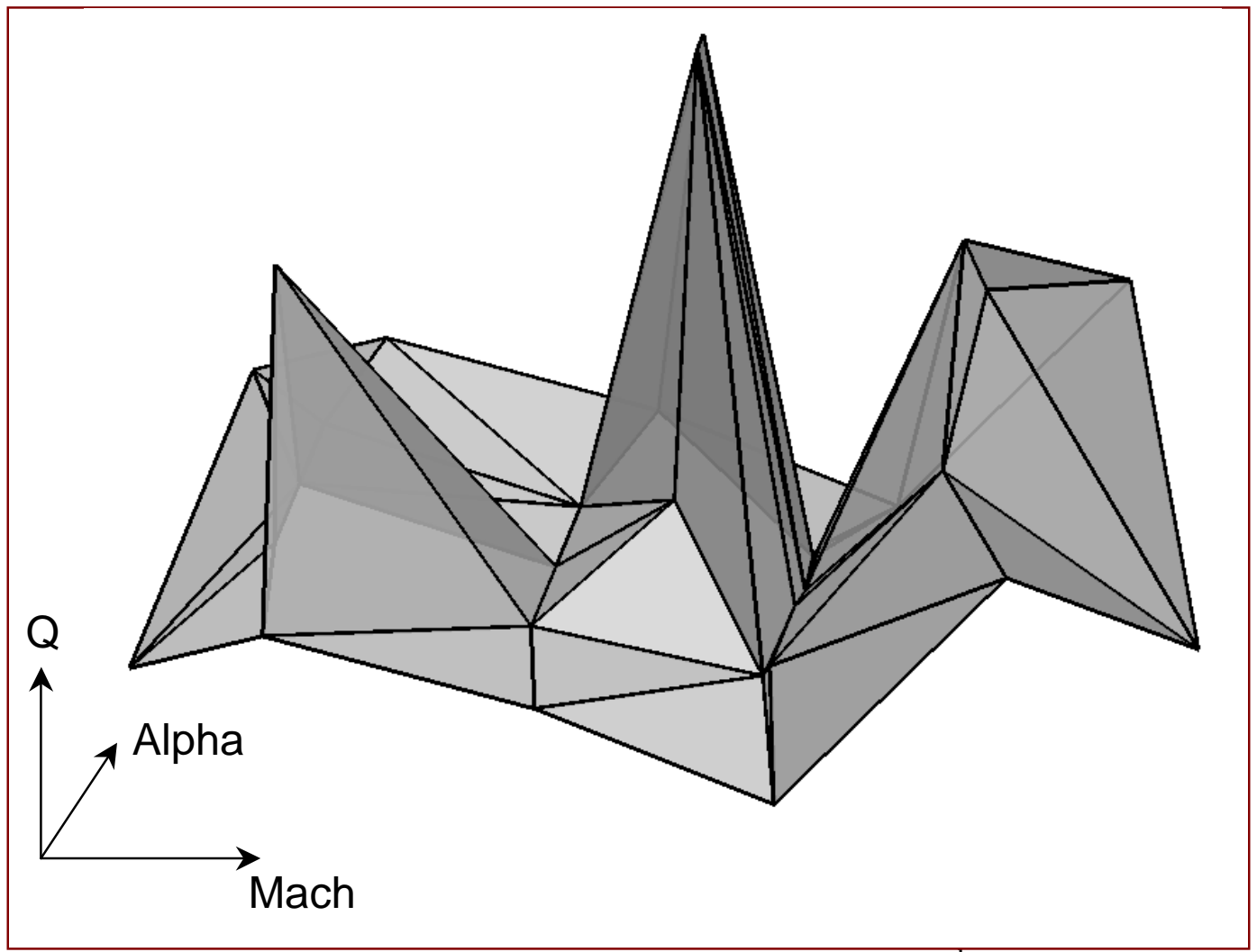

Fig. 22 "Two dimensional" tessellation of all the available CFD anchor points with the 3 rd dimension (dynamic pressure) shown. 


\begin{tabular}{|c|c|c|}
\hline Mach & Q, Bars & Alpha, degrees \\
\hline & & 147 \\
\hline 1.30 & 0.000001 & 148 \\
\hline 4.00 & 0.00001 & 149 \\
\hline 6.00 & 0.0001 & 150 \\
\hline 10.00 & 0.001 & 151 \\
\hline 12.50 & 0.010 & 152 \\
\hline 15.00 & 0.030 & 153 \\
\hline 17.00 & 0.065 & 154 \\
\hline 19.00 & 0.083 & 155 \\
\hline 20.40 & 0.100 & 156 \\
\hline 21.40 & 0.125 & 157 \\
\hline 23.00 & 0.150 & \\
\hline 25.00 & 0.175 & \\
\hline 26.00 & 0.200 & \\
\hline 27.00 & 0.250 & \\
\hline 29.00 & 0.290 & \\
\hline 30.00 & 0.325 & \\
\hline 31.00 & 0.350 & \\
\hline 32.20 & 0.400 & \\
\hline 34.00 & 0.475 & \\
\hline 36.00 & & \\
\hline 37.50 & & \\
\hline 40.00 & & \\
\hline 42.50 & & \\
\hline 45.00 & & \\
\hline 50.00 & & \\
\hline
\end{tabular}

Table. 2 Mach, Dynamic Pressure, and Angle of Attack conditions contained within the anchored CBAERO laminar and turbulent databases. The final tables are 'square' and thus contain $24 \times 20 \times 11=5280$ total cases. 


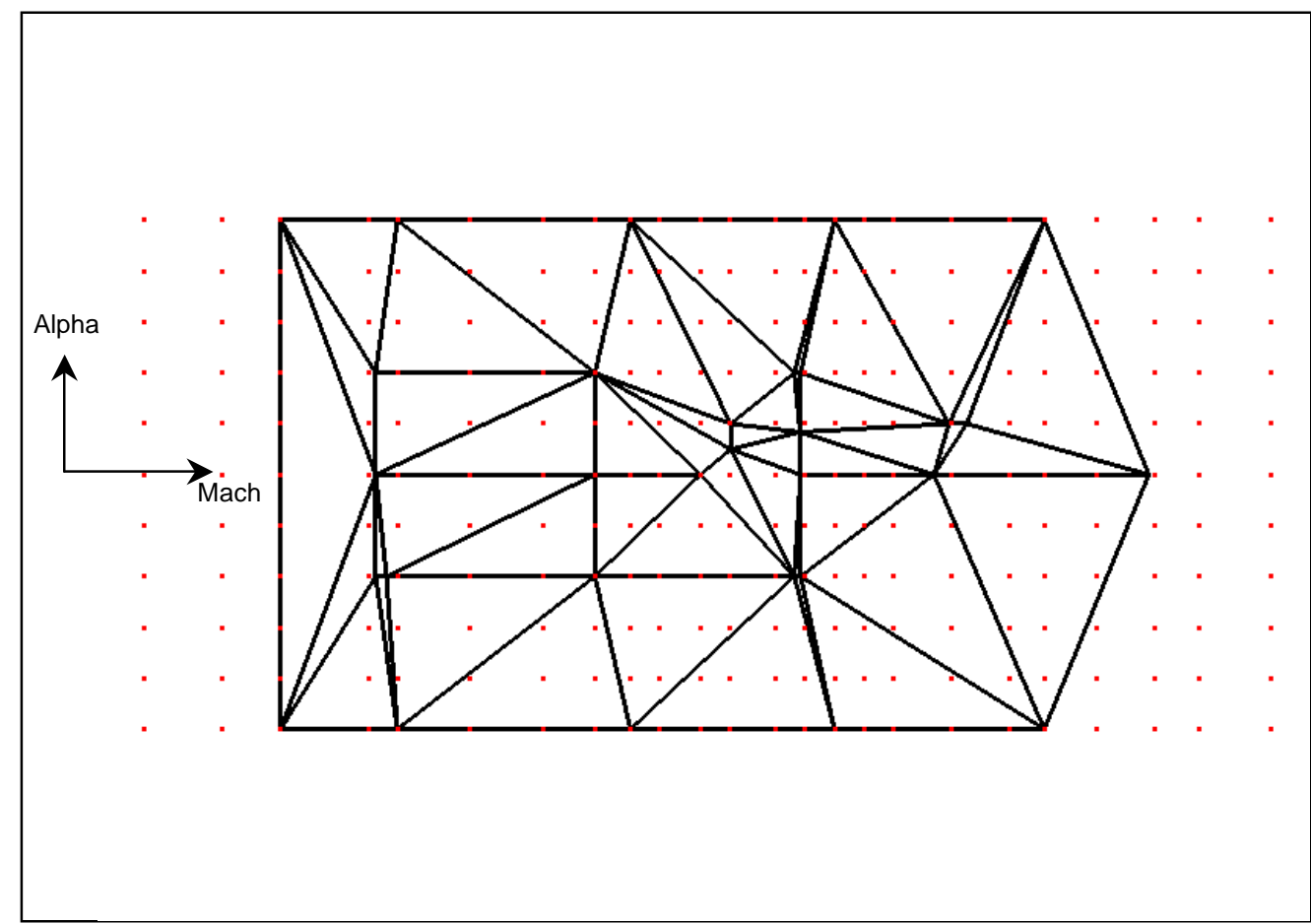

Fig. 23 "Two dimensional" tessellation of all the available CFD anchor points with the data base points (in red) shown.

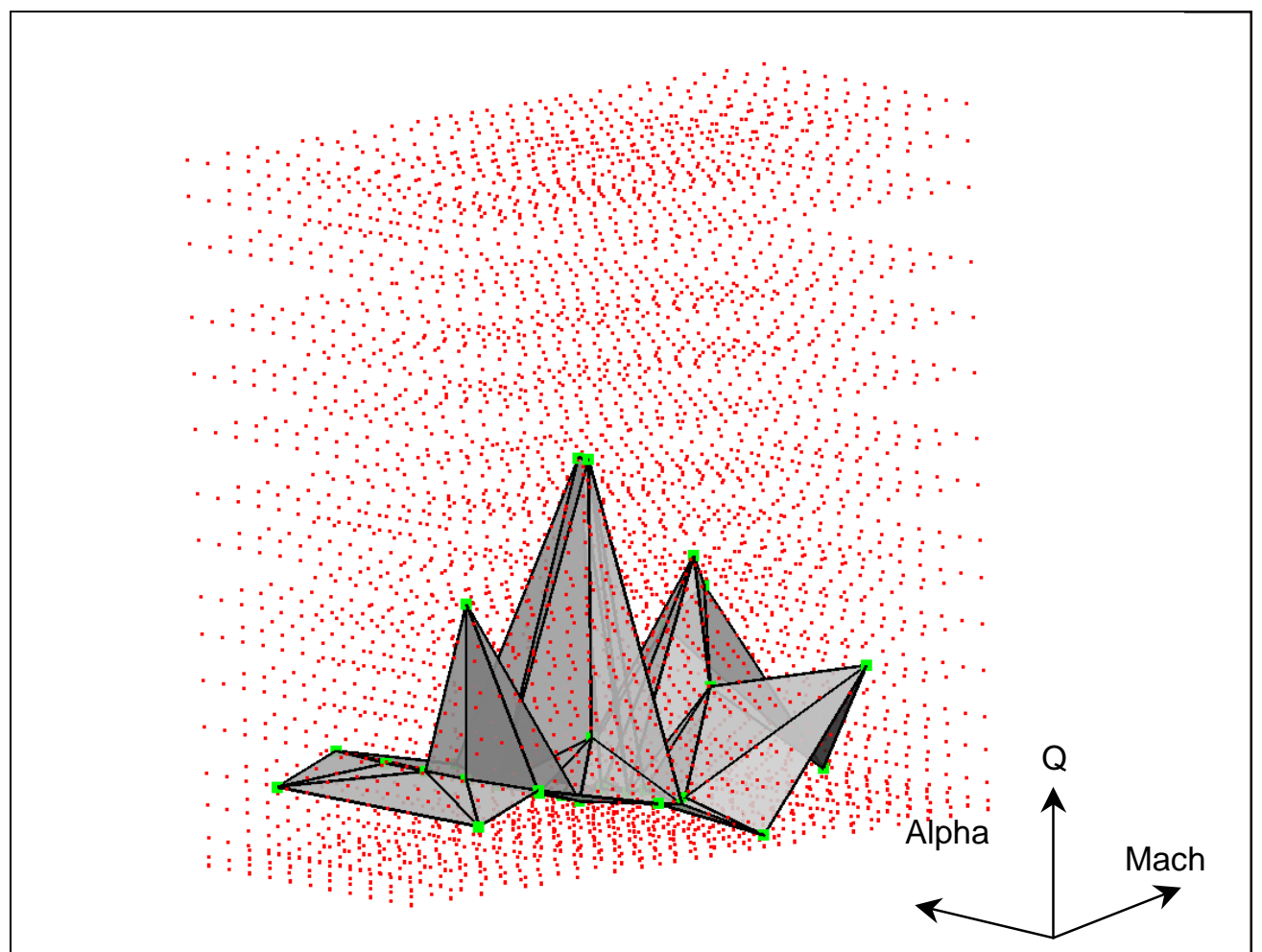

Fig. 24 "Two dimensional" tessellation of all the available CFD anchor points with the 5225 data base points (in red) shown in the full three dimensional Mach-Q-Alpha space. 


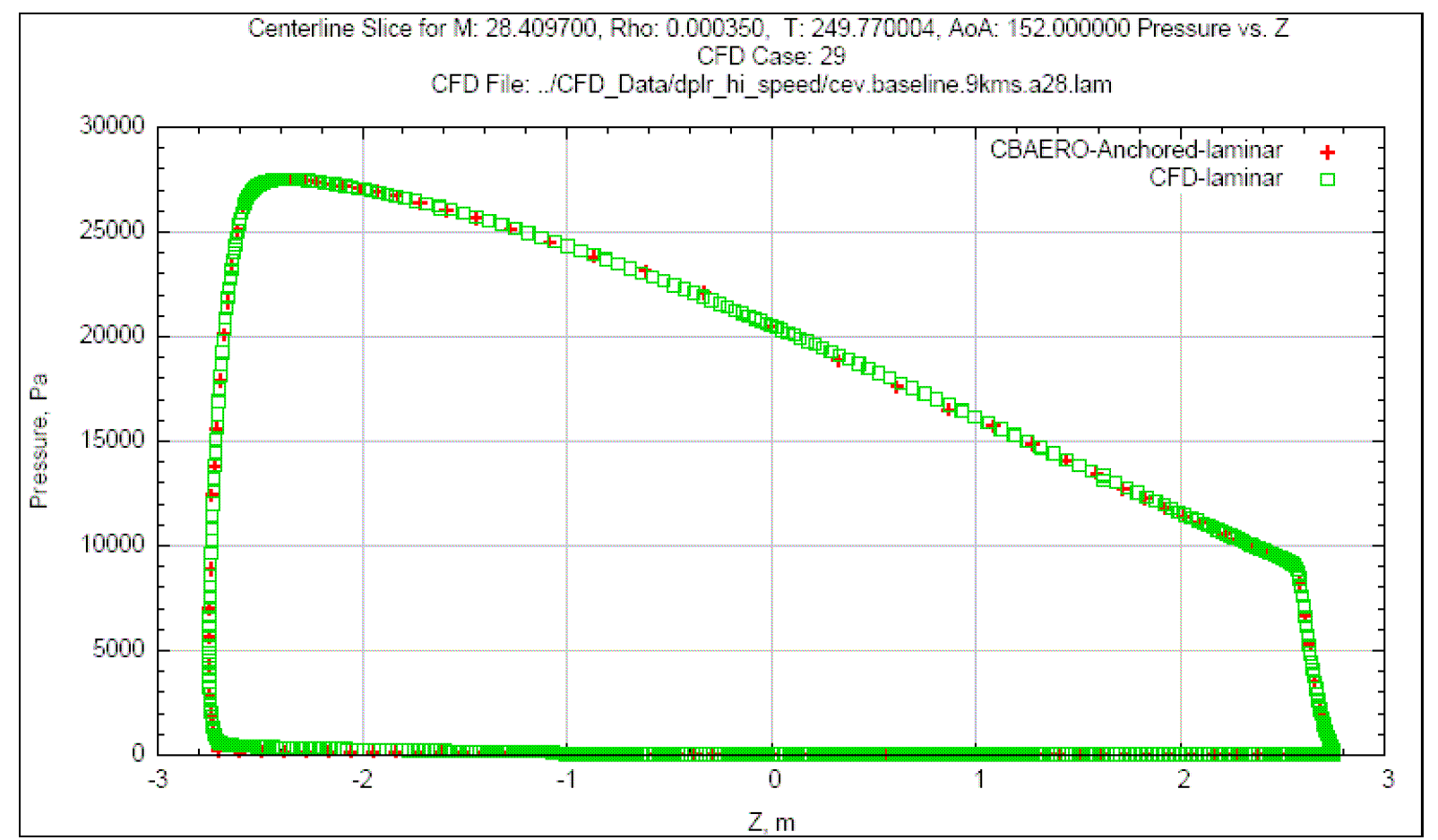

Fig. 25 Mach 28.4, 152.0 ${ }^{\circ}$ Angle of Attack, Centerline Pressure Distribution - Anchored CBAERO solution compared with DPLR results. All of the available CFD points were used in the anchoring process.

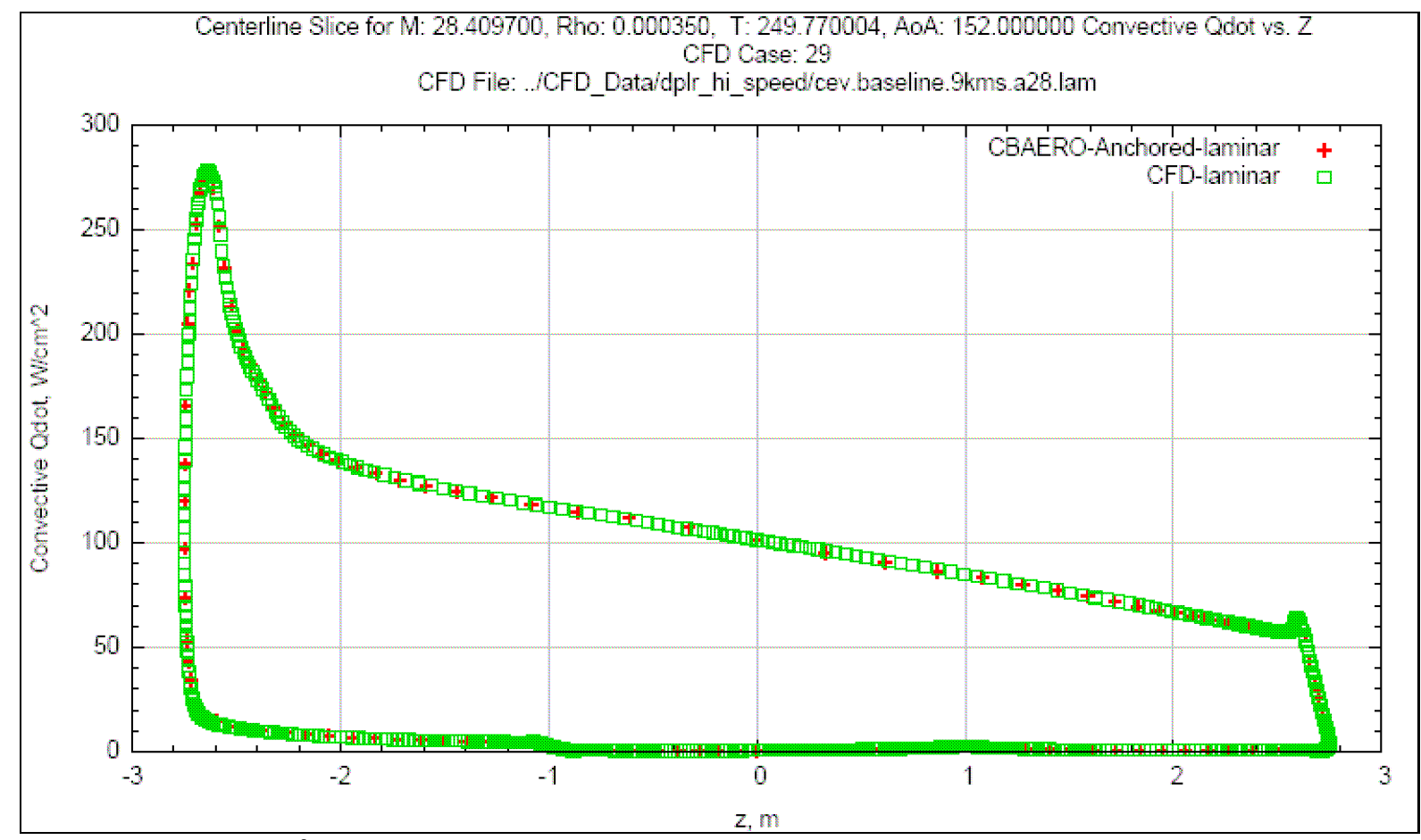

Fig. 26 Mach 28.4, 152.0 ${ }^{\circ}$ Angle of Attack, Centerline Laminar Convective Heating Distribution - Anchored CBAERO solution compared with DPLR results. All of the available CFD points were used in the anchoring process. 


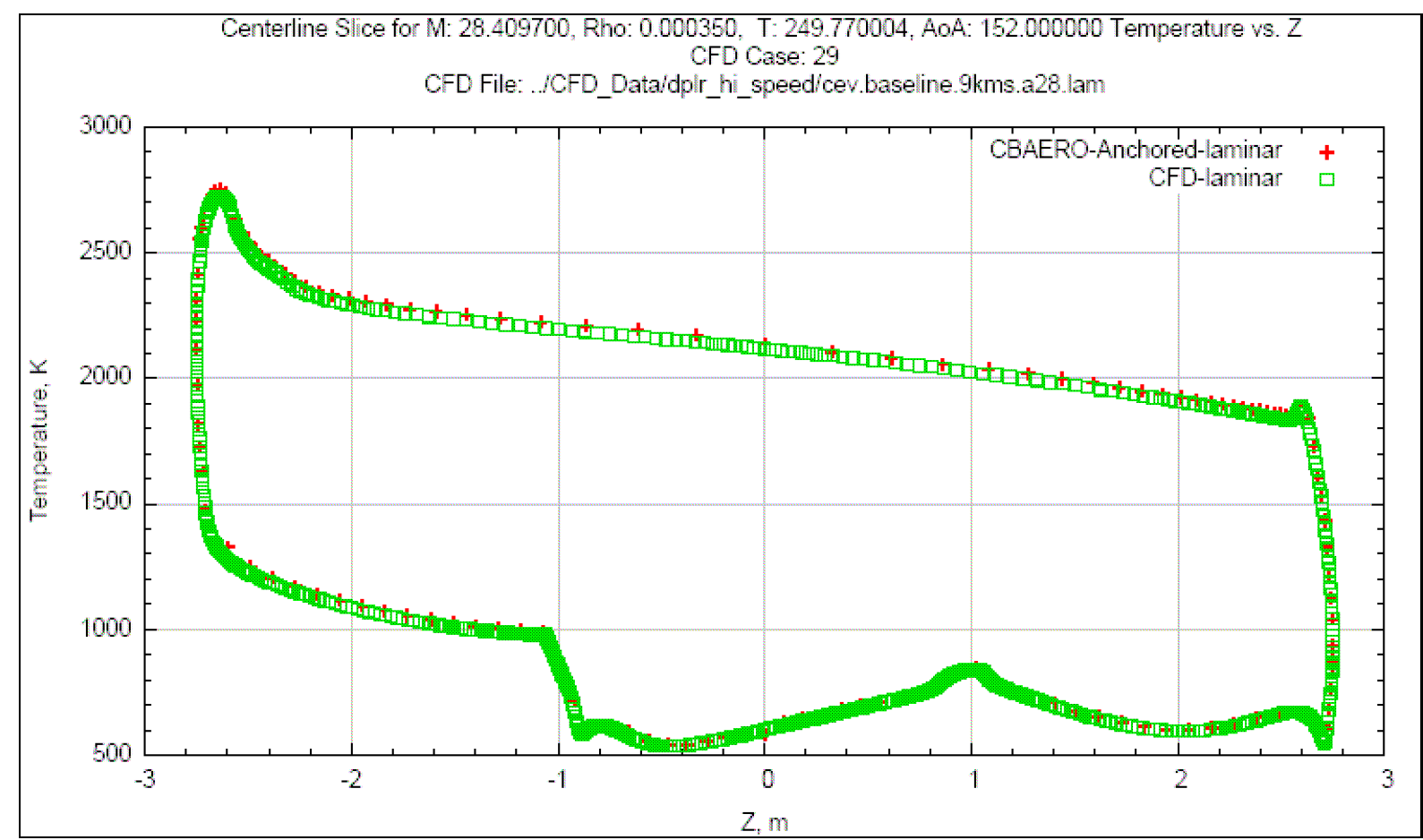

Fig. 27 Mach 28.4, 152.0 ${ }^{\circ}$ Angle of Attack, Laminar Temperature Distribution - Anchored CBAERO solution compared with DPLR results. All of the available CFD points were used in the anchoring process.

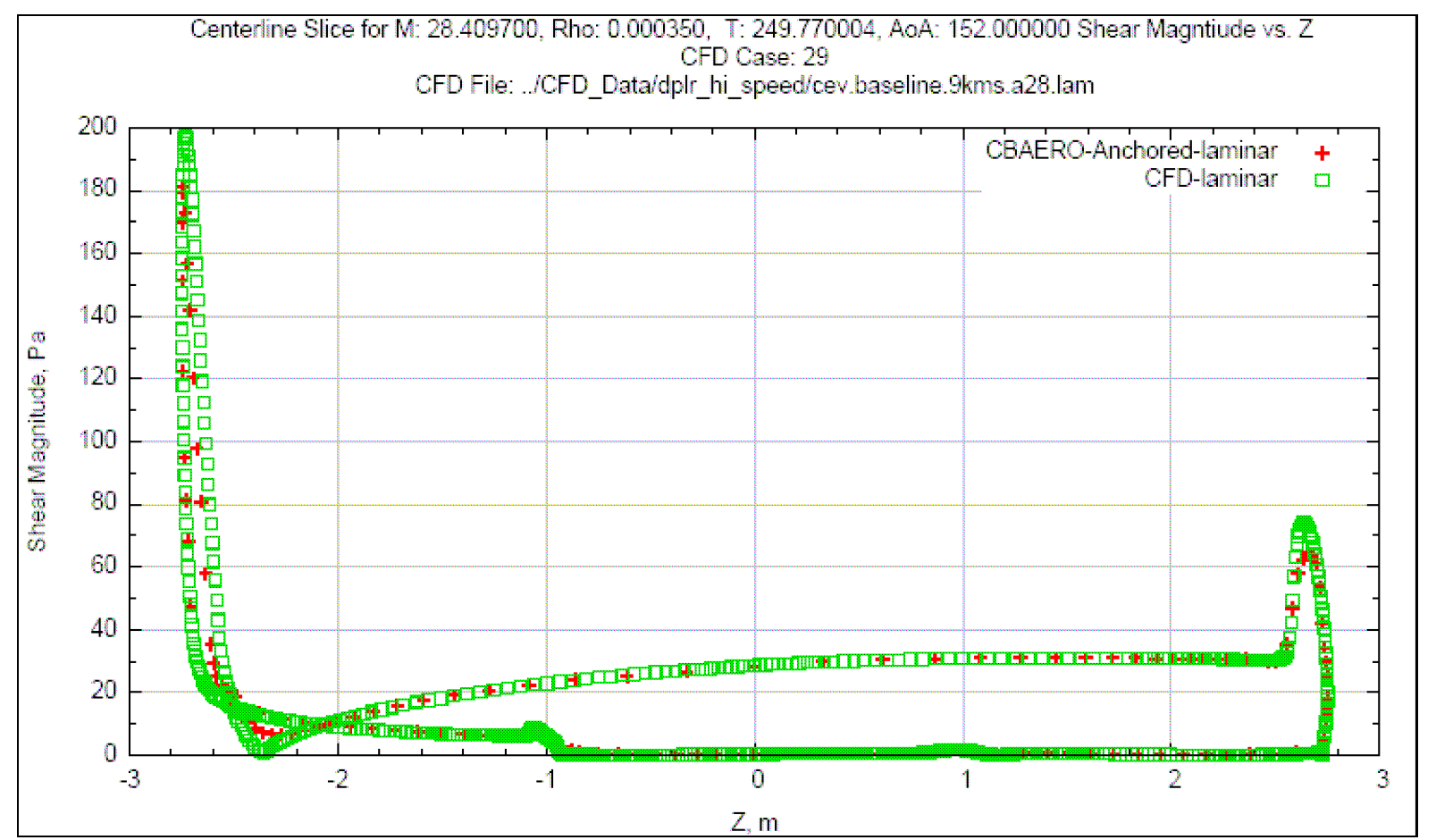

Fig. 28 Mach 28.4, 152.0 ${ }^{\circ}$ Angle of Attack, Centerline Laminar Shear Stress Magnitude - Anchored CBAERO solution compared with DPLR results. All of the available CFD points were used in the anchoring process. 


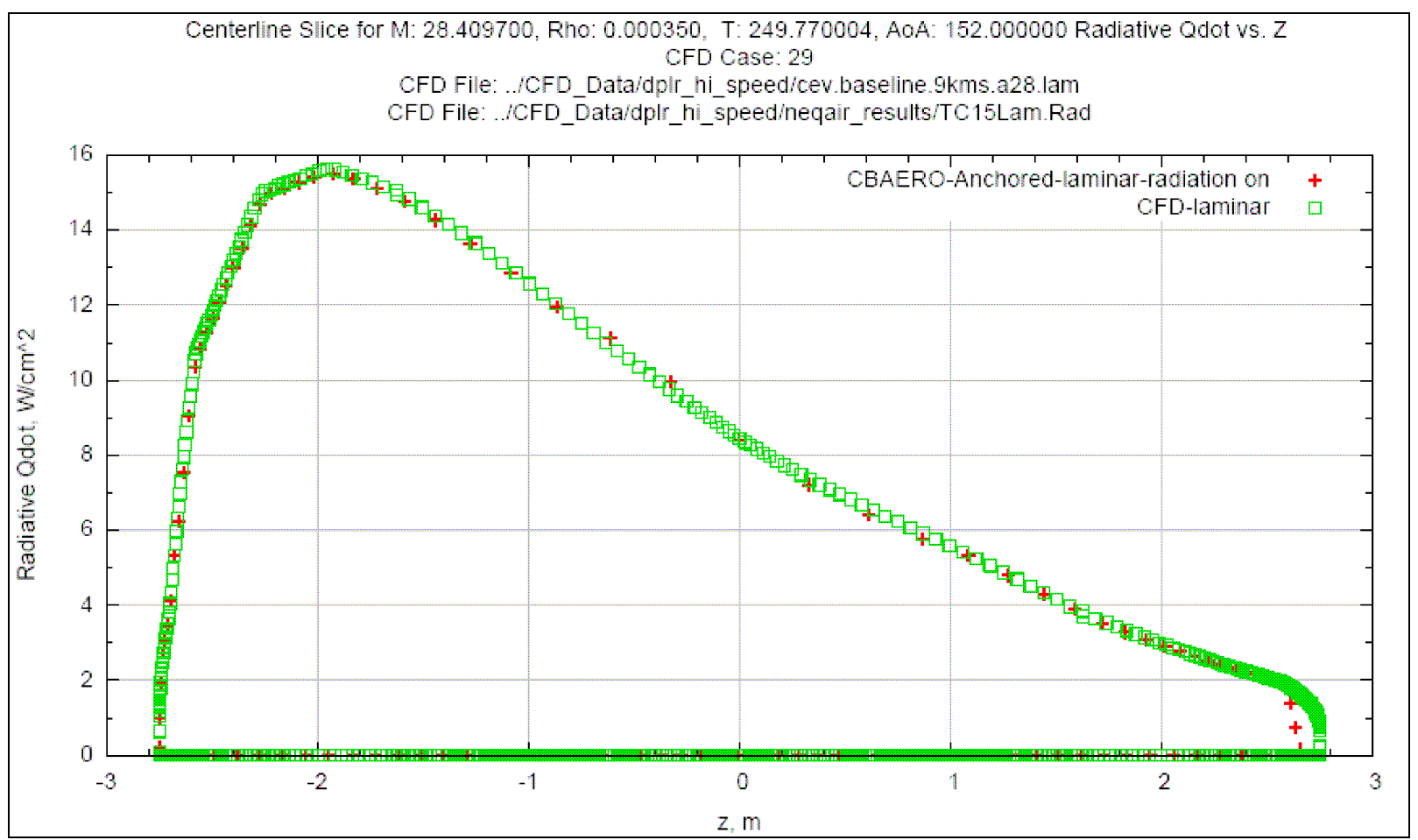

Fig. 29 Mach 28.4, $152.0^{\circ}$ Angle of Attack, Centerline Radiative Heating - Anchored CBAERO solution compared with NEQAIR results. All of the available CFD points were used in the anchoring process.

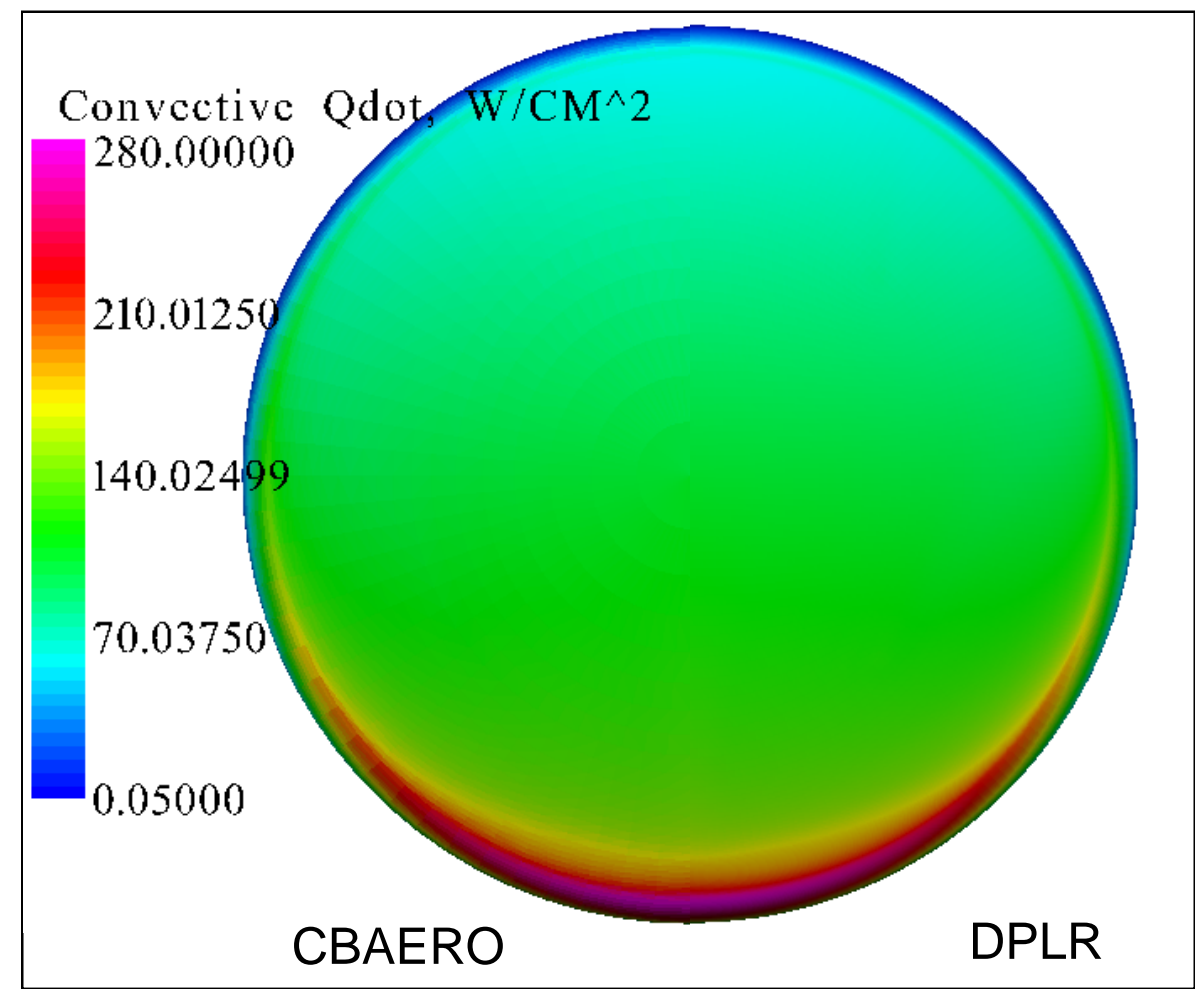

Fig. 30 Mach 28.4, $152.0^{\circ}$ Angle of Attack, Surface Laminar Convective Heating - Anchored CBAERO solution compared with DPLR results. All of the available CFD points were used in the anchoring process. 


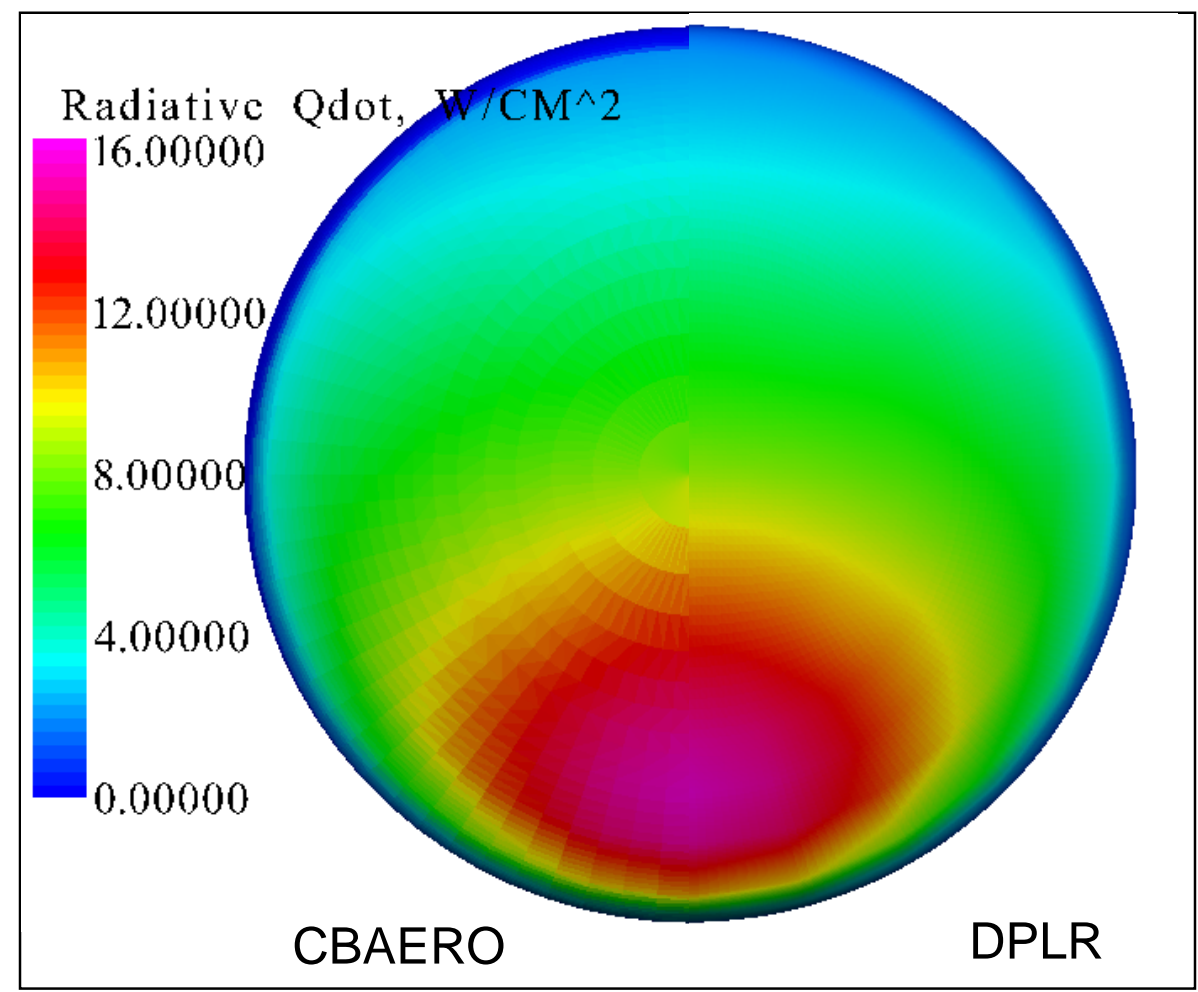

Fig. 31 Mach 28.4, 152.0 ${ }^{\circ}$ Angle of Attack, Surface Radiative Heating - Anchored CBAERO solution compared with DPLR results. All of the available CFD points were used in the anchoring process. 